University of Nebraska - Lincoln

DigitalCommons@University of Nebraska - Lincoln

Faculty Publications, Department of Psychology

Psychology, Department of

2007

Planning "Discrete" Movements Using a Continuous System: Insights from a Dynamic Field Theory of Movement Preparation

Anne R. Schutte

University of Nebraska-Lincoln, aschutte2@unl.edu

John P. Spencer

University of lowa

Follow this and additional works at: https://digitalcommons.unl.edu/psychfacpub

Part of the Psychiatry and Psychology Commons

Schutte, Anne R. and Spencer, John P., "Planning “Discrete" Movements Using a Continuous System: Insights from a Dynamic Field Theory of Movement Preparation" (2007). Faculty Publications, Department of Psychology. 492.

https://digitalcommons.unl.edu/psychfacpub/492

This Article is brought to you for free and open access by the Psychology, Department of at DigitalCommons@University of Nebraska - Lincoln. It has been accepted for inclusion in Faculty Publications, Department of Psychology by an authorized administrator of DigitalCommons@University of Nebraska - Lincoln. 


\title{
Planning "Discrete" Movements Using a Continuous System: Insights from a Dynamic Field Theory of Movement Preparation
}

\begin{abstract}
Anne R. Schutte and John P. Spencer
The timed-initiation paradigm developed by Ghez and colleagues (1997) has revealed two modes of motor planning: continuous and discrete. Continuous responding occurs when targets are separated by less than $60^{\circ}$ of spatial angle, and discrete responding occurs when targets are separated by greater than $60^{\circ}$. Although these two modes are thought to reflect the operation of separable strategic planning systems, a new theory of movement preparation, the Dynamic Field Theory, suggests that two modes emerge flexibly from the same system. Experiment 1 replicated continuous and discrete performance using a task modified to allow for a critical test of the single system view. In Experiment 2, participants were allowed to correct their movements following movement initiation (the standard task does not allow corrections). Results showed continuous planning performance at large and small target separations. These results are consistent with the proposal that the two modes reflect the time-dependent "preshaping" of a single planning system.
\end{abstract}

Key Words:

Studies of motor planning have recently emphasized the continuous, online nature of planning processes (Bastian et al., 1998; Bastian et al., 2003; Ghez et al., 1997; Favilla, 1997; Prablanc \& Martin, 1992; van Sonderen et al., 1989). This has been most dramatically shown in the timed-initiation paradigm used by Ghez and colleagues (1997). In this paradigm, participants are trained to initiate a movement when a "go" signal is given. The amount of time between the target onset and the go signal is varied, such that on some trials there is a very short time to plan a movement (e.g., $30 \mathrm{~ms}$ ) and on other trials there is a relatively long time to plan a movement (e.g., $250 \mathrm{~ms}$ ). Thus, this paradigm allows for an examination of the continuous evolution of a movement plan from a "default" response (essentially, a reasonable guess) to a fully planned and accurate movement.

The timed-initiation paradigm has revealed two modes of motor planning: continuous and discrete (Chou et al., 1999; Ghez et al., 1997; Favilla, 1997; Favilla, 2002; Ottes et al., 1984). In the continuous mode, people plan default responses at 
short planning times that are pointed toward the center of the target range or the weighted mean of the target range if the targets are not equi-probable (Favilla et al., 1990). As planning time increases, responses gradually shift toward the correct target. This mode occurs when targets are separated by less than $60^{\circ}$ (Ghez et al., 1997). In the discrete mode, people plan default responses at short planning times that are pointed toward one of the possible target locations. As planning time increases, the percentage of responses toward the correct target increases. This mode occurs when targets are separated by more than $60^{\circ}$ (Ghez et al., 1997).

Recently Erlhagen and Schöner (2002) proposed a new theory of movement planning, the Dynamic Field Theory (DFT). The DFT is a neural network model of movement planning that captures the continuous planning of hand kinematics as activation in a motor planning (MP) field. Activation in the MP field is distributed continuously over the space of movement parameters, such as movement direction, and activation evolves continuously through time according to a local excitation/lateral inhibition function. According to this function, neurons that code for similar movement directions co-excite one another, while neurons that code for very different directions inhibit one another. This allows the MP field to form stable, self-sustaining "peaks" of activation that reflect "decisions" about which direction to move to.

According to this model, initial biases such as those revealed in the timed-initiation paradigm reflect how the MP field is "preshaped" by three inputs. The first input is the task input, which captures perception of the possible target locations that are marked in the task space. The second input is the target input. This captures the presentation of the target at a particular location (i.e., direction) in the task space. Note that in the timed-initiation paradigm, the target is only presented for 30-230 ms. The third source of input to the MP field is a long-term memory (LTM) input. Results from several studies demonstrate that longer-term traces of past responses have an important influence on movement planning. For instance, Hening and colleagues (1988) showed that the default response in the timed-initiation paradigm can be shifted toward one target by increasing the frequency of responses to that target [for additional evidence of LTM effects on movement planning, see Erlhagen and Schöner (2002)].

To illustrate how these inputs "preshape" planning decisions in the MP field, consider the conditions central to the two modes account proposed by Ghez et al. (1997): the case where targets are close together $\left(<60^{\circ}\right)$ and far apart $\left(>60^{\circ}\right)$. Figure 1a shows a simulation of the DFT for a single timed-initiation trial with three marked, close targets $\left(20^{\circ}\right.$ apart $)$ in the task space and a brief presentation of a left target just before the "go" signal (see also, Erlhagen \& Schöner, 2002). The top layer in this simulation shows a Perceptual Input field (PI), the middle layer shows the Motor Planning field (MP), and the bottom layer shows the Long-Term Memory field (LTM). The $x$-axis within each layer shows a collection of directionally-tuned neurons arranged by their "preferred" movement directions (for a detailed discussion of ties to neurophysiology, see Erlhagen et al., 1999; Jancke et al., 1999). The $y$-axis shows the activation of each neuron. The $z$-axis captures time from the start of a trial ( $0 \mathrm{~ms}$; back of figure) to the end of a trial (front of figure). ${ }^{1}$

PI (see top layer of Figure 1a) is a simple input field (this field has no interactive neural dynamics) that brings together (i.e., sums) the two visible inputs in the timed-initiation task-the task and target inputs. The large Gaussian input toward 

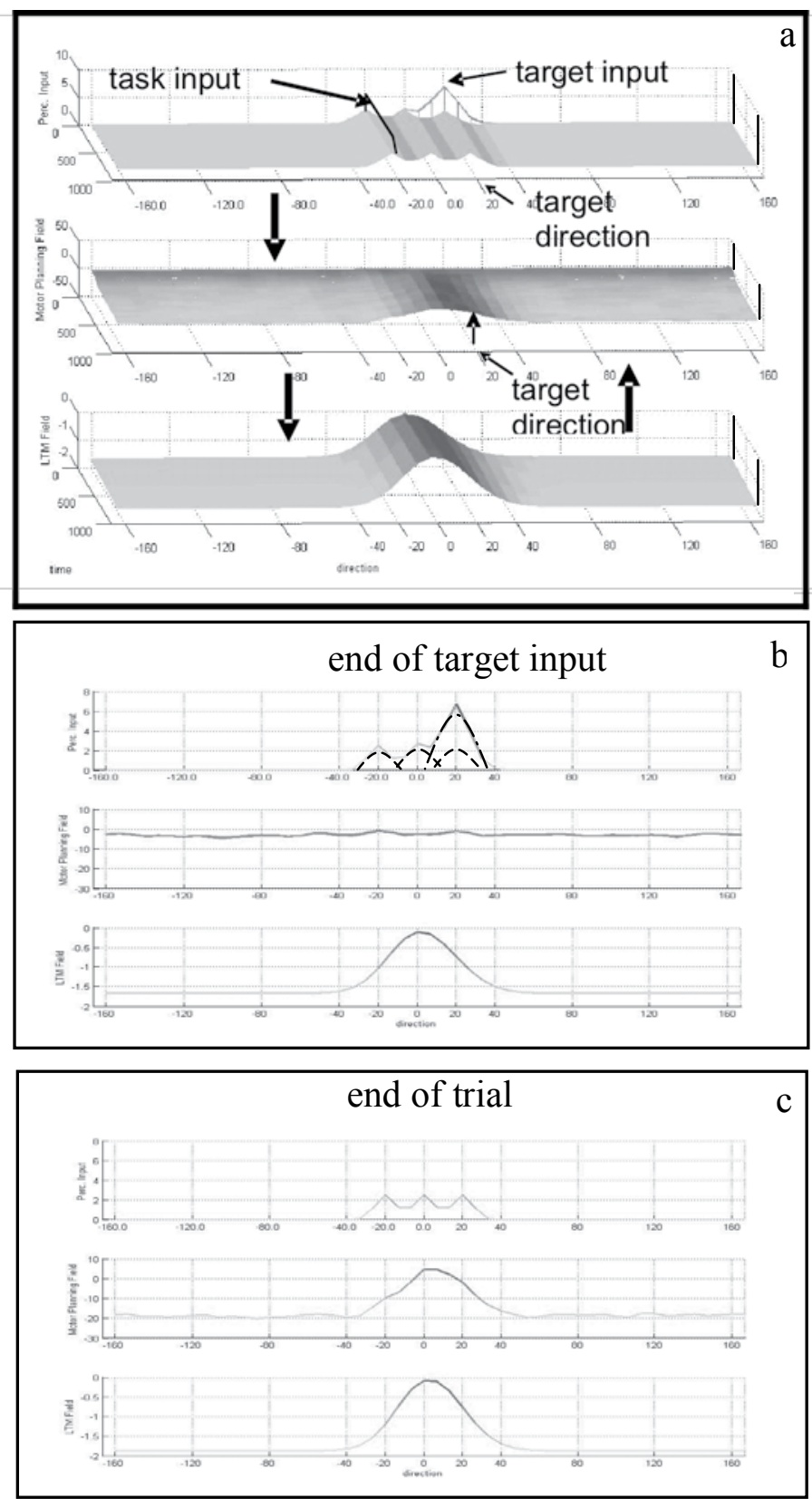

Figure 1-Simulations of the DFT with a small target separation. Panels a-c are with a short planning time, and panels $\mathrm{d}$-f are with a long planning time. Location is on the $x$-axis, activation is on the $y$-axis, and time is on the $z$-axis. The top layer in each panel is perceptual input, the center layer is the motor planning field, and the lower layer is the longer-term memory field. See text for further details. 

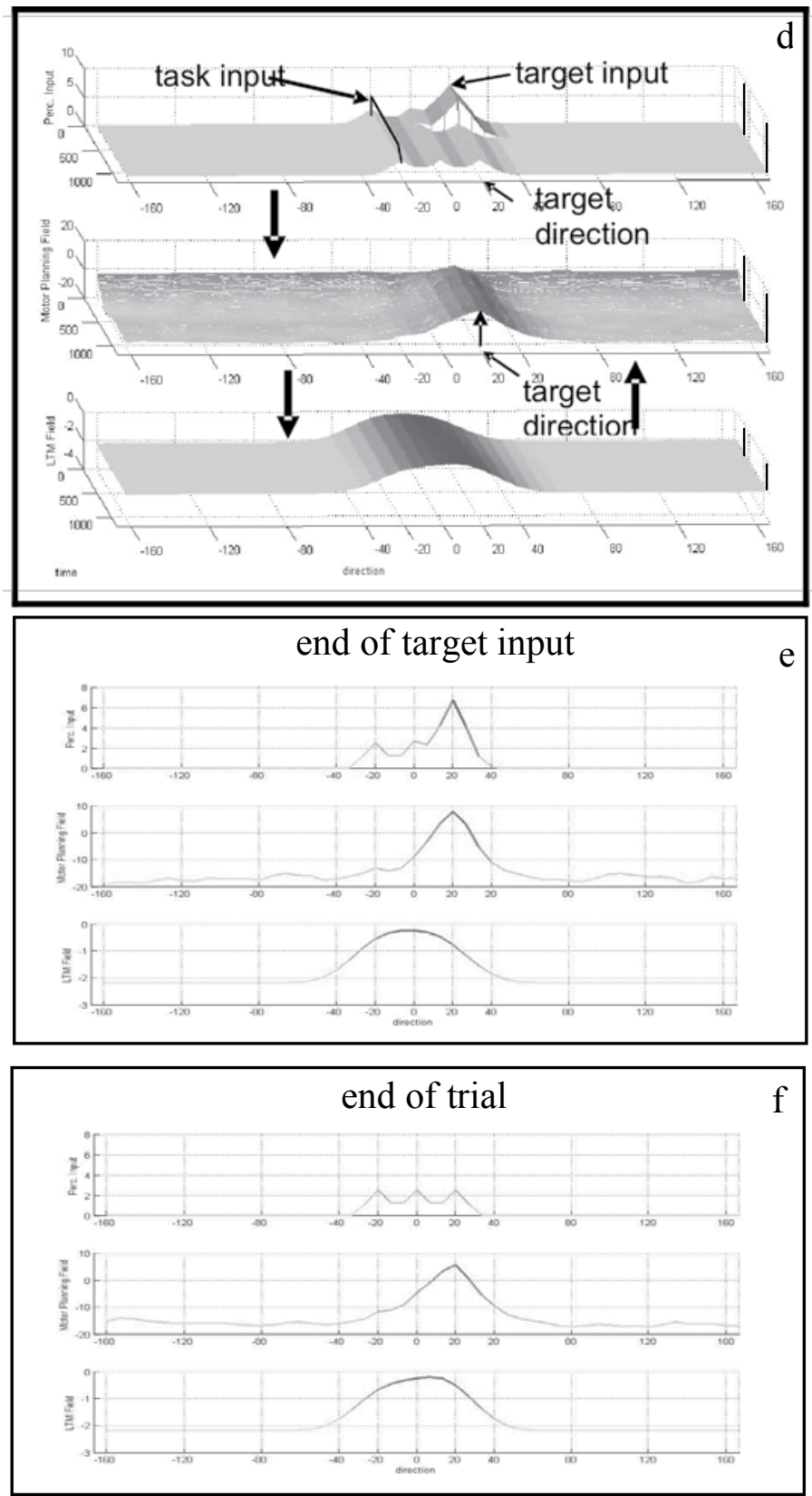

Figure 1, continued 
the back of the simulation reflects the presentation of the target for $30 \mathrm{~ms}$ at $-20^{\circ}$ in the task space. The smaller distributions of activation in PI toward the front of the simulation reflects the task input resulting from three marked locations in the task space at $-20^{\circ}, 0^{\circ}$, and $20^{\circ}$ (note that $0^{\circ}$ in these simulations reflects a straight ahead movement direction with negative targets to the left, that is, counterclockwise).

To get a better feel for the inputs in PI (as well as the dynamics of MP and LTM; see below), we show two time slices through the simulation in Figure 1a in the figures below (at the times marked by the vertical lines to the right of each simulation panel). Figure $1 \mathrm{~b}$ is a cross-section from the last millisecond of the target presentation (at $30 \mathrm{~ms}$ ). At this point in time, the input to PI (see top layer in Figure $1 b$ ) is captured by three Gaussian inputs indicated with dashed lines-one for each marked possible target in the task space-and the target input which is a larger Gaussian input indicated with a dot-dashed line. Because the possible targets are close together $\left(20^{\circ}\right.$ separation), the activation profile produced by these inputs blend together (see solid line in the top layer of Figure 1b). The final time slice in Figure 1c shows activation in the model at the end of the trial (at $780 \mathrm{~ms}$ ). As can be seen in the top panel of Figure 1c, the only perceptual input still "on" at the end of the trial is the task input.

The second layer in Figure 1a shows the evolution of neural activation in the MP field during the course of a single trial. Toward the beginning of the trial, the MP field is relatively homogeneous (see middle layer of Figure 1b) because the task input (top layer) and the LTM input (bottom layer) are relatively weak, and the target input was too brief to build an activation peak. Even though the target is centered at $-20^{\circ}$, a peak forms in the MP field near $0^{\circ}$ (Figure 1c, center layer). This occurs for three reasons. First, the MP field was "preshaped" around $0^{\circ}$ by the LTM input. Second, the target input duration in this simulation was quite short (30 $\mathrm{ms}$ ); thus, this input had little chance to dominate activation in the MP field. Third, the target input overlaps with the task and LTM inputs because the possible targets are close together. Thus, the onset of the target input boosts activation both around the target location $\left(-20^{\circ}\right)$ but also around the preshaped location $\left(0^{\circ}\right)$. Because the preshaped neurons around $0^{\circ}$ were closer to threshold (i.e., closer to 0 ), the boost from the target input - though weaker than at $-20^{\circ}$ - was sufficient to drive the formation of a stable activation peak in MP. The model would "respond" at the end of the trial by initiating a movement directed to $0^{\circ}$ - the location of maximal activation at the end of the simulation.

The final layer in Figure 1a shows the LTM field. Activation in the LTM field reflects traces of past decisions made by the MP field. In particular, peaks of activation in the MP field leave activation traces at the associated directions in the LTM field. Conversely, activation in the LTM field is fed into the MP field as input (see arrows connecting the central and bottom layers in Figure 1a). Note that activation in the LTM field evolves over a relatively slow time scale. Consequently, activation traces in this field grow slowly and decay slowly (for details, see Erlhagen \& Schöner, 2002). In Figure 1a, the LTM field has a uni-modal distribution of activation centered at $0^{\circ}$ (the center target; see also bottom layer of Figure $1 \mathrm{~b}$ ). This activation profile was constructed by running the model through 12 trials with a $30 \mathrm{~ms}$ target presentation time and randomly presenting the target at either the left $\left(-20^{\circ}\right)$, center $\left(0^{\circ}\right)$, or right $\left(20^{\circ}\right)$ target on each trial. Note that because the MP field selected the center target in this simulation, there is little change in LTM 
during the course of the trial (there is a small increase in activation strength at $0^{\circ}$; compare the bottom layers of Figures $1 \mathrm{~b}$ and 1c).

Figures 1d-f show how the same model responds on a trial with a longer planning time, in this case, with the target input "on" for $300 \mathrm{~ms}$. As can be seen in Figure 1e, by the end of the target presentation period $(300 \mathrm{~ms})$ the model has built a peak in the MP field centered at the target location $\left(20^{\circ}\right)$. Consequently, at the end of the trial the point of maximal activation in the MP field is centered over the actual target direction and the model generates an accurate response. Note that the LTM in this simulation is broader (compare the bottom layers in Figure $1 \mathrm{~b}$ and 1e). This is due to more accurate responses to the left and right target locations on previous trials. For instance, there is a clear distortion in LTM toward the right target by the end of the simulation (see bottom layer of Figure 1f).

What about the second condition central to the two modes account-how does the DFT behave when the targets are far apart? When target locations are far from each other at $-80^{\circ}, 0^{\circ}$, and $80^{\circ}$, the task input is tri-modal: there are three individual activation profiles that reflect perception of the three marked targets in the task space (see top layers of Figure $2 \mathrm{a}$ and $2 \mathrm{~d}$ ). Similarly, activation in the LTM field is tri-modal, reflecting that on past trials, the model always moved to one target or the other and never in between the marked locations (see lower layers of Figure $2 \mathrm{a}$ and $2 \mathrm{~d}$ ). This "discrete" form of responding is captured by the behavior of the MP field in Figure 2a. In this simulation, the target was presented for a short duration $(30 \mathrm{~ms})$ as was the case in Figure 1a. With this short planning time, the model "guesses" to the center target (see center layers of Figures $2 \mathrm{a}$ and $2 \mathrm{c}$ ). This occurs for two reasons: (1) the target duration was quite short, allowing little opportunity for the target input to strongly shape activation in the MP field; and (2) spontaneous fluctuations (i.e., noise) helped boost activation near the center target.

At a longer planning time, by contrast, the target input dominates activation in the MP field and the model plans a movement to the correct target. This is shown in Figure 2d. In this simulation, the target was "on" for $300 \mathrm{~ms}$ (as in Figure 1d). As in Figure 1d, a peak builds at the correct target location by the end of the target presentation (see Figure 2e, center layer), and remains centered at the target location until the end of the trial (see Figure $2 \mathrm{f}$, center layer). Note that this occurs even though activation in the LTM field is biased toward the center and right targets during target presentation (see bottom layer of Figure 1e).

\section{Overview of the Present Study}

The present study tested conflicting predictions of the two modes and DFT accounts of performance in the timed-initiation paradigm. According to the two modes account, the planning mode is dictated primarily by the target separation (Ghez et al., 1997; Favilla, 2002). By contrast, according to the DFT, continuous-looking versus discrete-like performance depends on the details of the task, target, and LTM inputs. Here, we attempted to create a more continuous-looking response distribution in a situation where targets were far apart by manipulating two aspects of the standard timed-initiation paradigm.

First, participants completed the task in a homogeneous task space with unmarked locations. This should eliminate the task input, leaving the LTM input as 

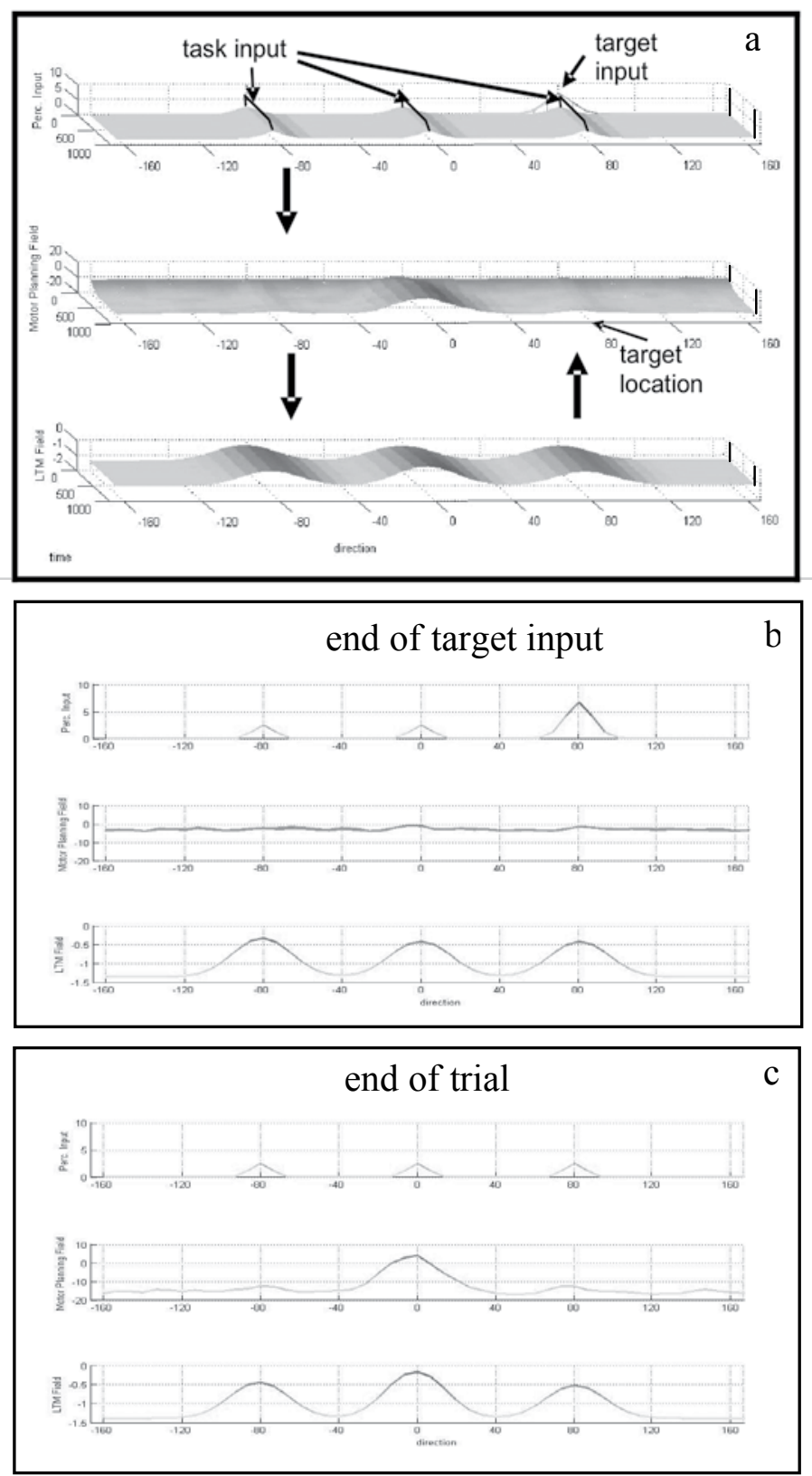

Figure 2-Simulations of the DFT with a large target separation. Panels a-c are with a short planning time, and panels $\mathrm{d}$-f are with a long planning time. Location is on the $x$-axis, activation is on the $y$-axis, and time is on the $z$-axis. The top layer in each panel is perceptual input, the center layer is the motor planning field, and the lower layer is the longer-term memory field. See text for further details. 

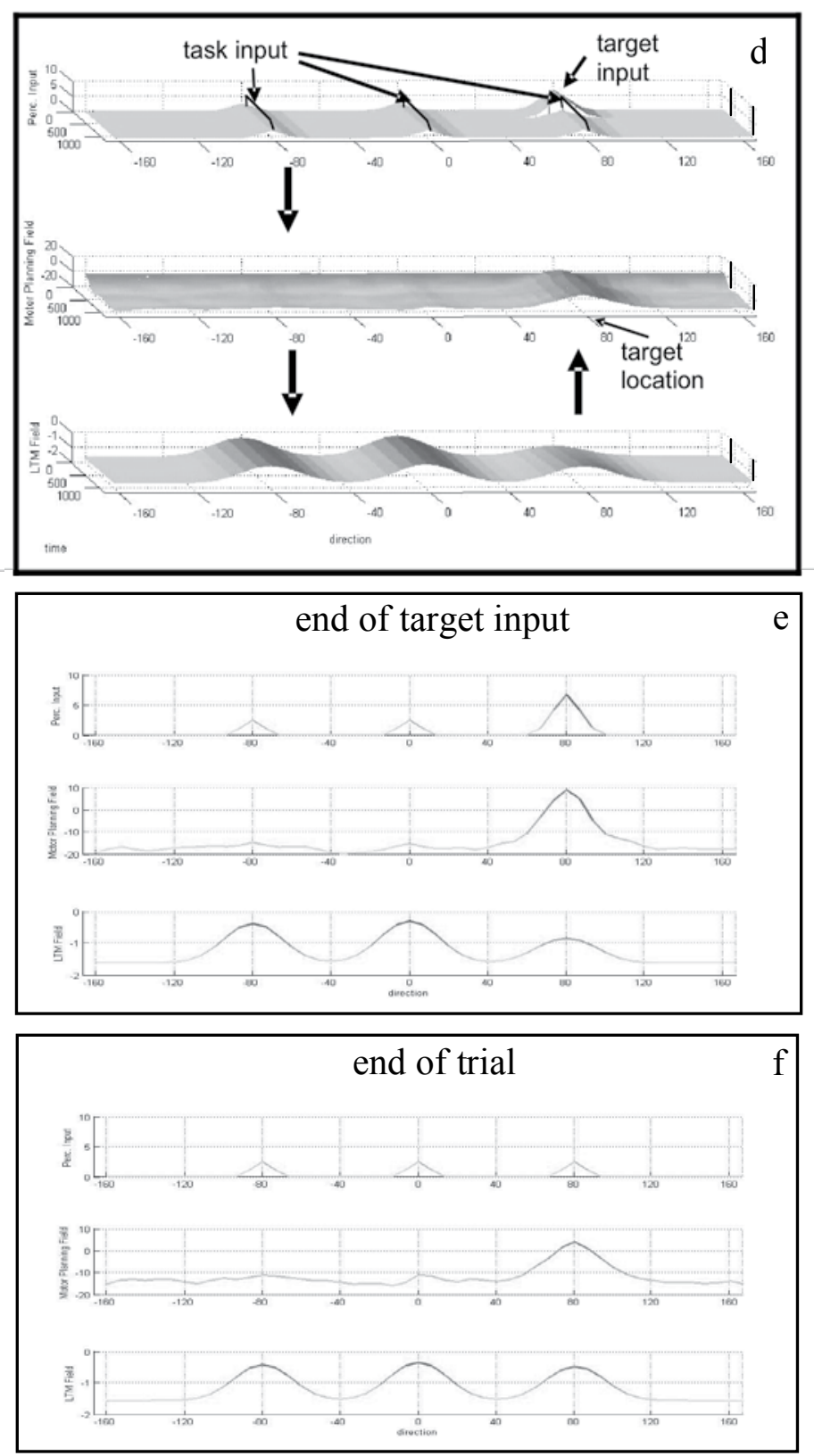

Figure 2, continued 
the sole "preshaping" influence on movement planning. Second, we allowed people to correct their movements on-the-fly during each trial. In the standard paradigm, participants are instructed to make straight-line movements toward a single target location on each trial without corrections. Based on the mechanism that creates the LTM input in the DFT - activation in the MP field leaves traces of activation in LTM-we hypothesized that allowing corrections would "smear out" activation in the LTM field because movements to multiple locations would be planned in the MP field on trials with short planning times (e.g., the initial default plan followed by a potentially more accurate corrective plan). If our two manipulations result in a more distributed pattern of activation in LTM, then responses at short planning times should look continuous, that is, responses should be distributed across the target range with many responses in between the actual target locations. Importantly, this should occur even when the targets are far apart $\left(>60^{\circ}\right)$.

We discuss these two experimental manipulations - and our view of the link between the motor planning system captured by the DFT and the control of movement trajectories - in greater detail in the introduction to Experiment 2. Before turning to these issues directly, however, it was important to replicate the central effects from Ghez et al. (1997) in the task setting used in the present study.

\section{Experiment 1}

Given that the central goal of this study required that we modify the timed-initiation paradigm, we first attempted to replicate the findings from Ghez et al. (1997) using our task setting. Note that we used the instructions from Ghez et al., that is, movement corrections were not allowed in the present experiment. This contrasts with the critical manipulation in Experiment 2.

There were four main differences between our task context and the one used by Ghez and colleagues (1997). First, the target locations were not marked. As described previously, this should leave two inputs to the MP field-the LTM input and the target input. Note that Favilla (2002) has used the timed-initiation paradigm in tasks without marked target locations and has replicated and extended the original Ghez et al. findings.

The remaining three modifications of the task were meant to bring the timedinitiation paradigm into register with our previous work on the dynamics of spatial working memory (see, e.g., Schutte et al., 2003; Spencer \& Hund, 2002; Spencer \& Schöner, 2003; Spencer et al., in press). Thus, movement distance was always $15 \mathrm{~cm}$ (vs. 3.2-38.4 cm in Ghez et al.). Next, we included three targets in the task space (vs. two in Ghez et al.): one to the left of the midline of the task space, one aligned with the midline axis, and one to the right of midline. It is important to note that the DFT makes the same predictions whether there are two or three targets: when two targets are close together, the activation patterns associated with the targets will blend in the LTM field and responses will be directed toward the average of the two targets at short planning times; when the targets are far apart, the activation patterns associated with the targets will remain isolated in LTM and responses will be directed toward one target or the other at short planning times. The final difference was that participants were allowed full-view of their hand (view of the hand was occluded in Ghez et al.). Thus, participants operated in a 
closed-loop rather than open-loop mode. This means that it was possible for participants to visually correct their movements based on their memory of the target location, despite our instructions.

Overall, we did not expect these modifications to significantly impact the results, that is, we expected to replicate the continuous vs. discrete findings from Ghez et al. (1997). Nevertheless, given the many changes between our task setting and the original context in which the timed-initiation paradigm was developed, this study provides an important test of the robustness of the original findings.

\section{Method}

\section{Participants}

A total of 14 adults ( $M=18.8$ years; $S D=0.67$ years) participated in this study. Participants were recruited from the undergraduate and graduate student body and staff at the University of Iowa. Participants received either course credit or payment for their participation. All participants were right-handed. The number of females and males was roughly balanced across conditions. This study was approved by the ethics committee of the University of Iowa, and it was performed in accordance with the ethical standards laid down in the 1964 Declaration of Helsinki. All participants gave their informed consent before participating in the study.

\section{Materials}

Participants sat at a $0.92 \mathrm{~m} \times 1.19 \mathrm{~m}$ horizontal table, the top of which was a smooth black surface. An arc was removed from one side of the table, and participants were seated in an adjustable chair positioned within this arc with the table top at belly height. The lights in the room were dimmed and black curtains were hung across the ceiling and extended to the floor to prevent reflections from appearing on the table top. After these adjustments, the table top appeared to be a smooth, homogenous surface. A Barco 708 rear-projection system was used to project the target images and start location on to the table's surface. Images were projected at $60 \mathrm{~Hz}$ at a resolution of $1,024 \times 768$ pixels. Targets were equilateral triangles (which we called "spaceships" in the task) with a base of $13 \mathrm{~mm}$. Targets were always $15 \mathrm{~cm}$ from the starting location. The starting location was a small yellow circle (18 mm radius). A personal computer controlled the type and timing of all stimuli presented in the experiment using customized software.

Participants' movements were recorded using an Optotrak motion analysis system (Northern Digital, Inc.). Optotrak tracks small (radius $=3.5 \mathrm{~mm}$ ), individually pulsed infrared emitting diodes (IREDs) within a specified three-dimensional volume with better than $1 \mathrm{~mm}$ precision. IRED data were collected in two dimensions at $150 \mathrm{~Hz}$ and then converted to 3-D coordinates using the Direct Linear Transformation technique. Three IREDs were placed on the participant's index finger to ensure good IRED visibility. The IRED placed directly above the fingernail always had the best visibility, so data from this IRED were used in all analyses. 


\section{Task and Procedure}

Each trial began when the computer said, "Beginning search for enemy spaceships." After a random pre-trial delay ranging from 2-4 s, the computer played a "ready, set, go" sequence. The target was illuminated 30, 80, 130, 180, or $230 \mathrm{~ms}$ before the end of this sequence. The target was turned off at the end of "go" or at movement initiation if the participant moved their finger before the end of "go." Participants were instructed to slide a small disk along the surface of the table to where they thought the "spaceship" was hiding when they heard "go." Movement speed was not emphasized, but initiation time relative to the "go" signal was. Participants were also instructed to keep their movements as straight as possible, even if the movement was in the wrong direction (see Ghez et al. 1997). At the end of the movement, they were asked to remain at that position until the computer displayed the feedback screen. After $3.5 \mathrm{~s}$, the target was re-illuminated for $1.5 \mathrm{~s}$ ( $2 \mathrm{~s}$ during the practice phase of session one) so the participant could compare the location of their finger with the actual target location.

Next, four types of feedback information were displayed on the table for 2 s: (1) initiation time relative to the "go" signal; (2) the sum of an accuracy score and initiation time score; (3) the total accumulated points; and (4) a "flight rank." Initiation time scores ranged from 5 points for responses begun within $\pm 40 \mathrm{~ms}$ of the "go" signal to 0 points for responses begun more than $200 \mathrm{~ms}$ before/after "go." Accuracy scores ranged from 5 points for responses $0-1 \mathrm{~cm}$ from the target to 0 points for responses $3 \mathrm{~cm}$ or greater from the target. Point scores of 10 and 9 were accompanied by a "Direct Hit!" and "Good Job!" message, respectively. The computer warned participants if their initiation times were at the boundaries of the acceptable initiation time range ( $\pm 200 \mathrm{~ms}$ ). Participants received one new star for each rank. Promotions occurred every 160 points. After feedback, the table was blanked, there was a short delay, and the computer began the next trial.

\section{Experimental Design}

Participants were randomly assigned to one of two experimental conditions. All participants moved to three target locations-a left, center, and right location. The separation between targets varied across conditions. In condition 1, adjacent targets were $20^{\circ}$ apart, and in condition 2, adjacent targets were $80^{\circ}$ apart. Participants responded to target locations at five different planning times (i.e., 30, 80, 130, 180, and $230 \mathrm{~ms}$ before the end of "go!"). In each condition, participants completed four types of trials. Participants completed a number of practice trials at the start of each experimental session (15 at the start of session 1, 6 at the start of session 2). These ensured that participants understood the task and feedback messages. After practice, participants completed a block of simple trials. These trials were identical to the standard planning trials expect that participants knew the order in which the targets would appear (see Ghez et al. 1997). Next, participants completed a block of choice trials in which the location of the target on each trial was unpredictable. Finally, both simple and choice trials contained control trials in which the spaceship remained illuminated before, during, and after the "ready, set, go!" sequence. These trials were examined to determine that the apparatus was calibrated correctly-i.e., the participants' responses matched the target locations. Only data 
from the choice trials were analyzed for the present report. Participants completed 8 trials to each target at each planning time across two experimental sessions, for a total of 120 choice trials.

\section{Method of Analysis}

Three-dimensional motion data were filtered using a second-order recursive low pass Butterworth filter (Winter, 1990) with a $20 \mathrm{~Hz}$ cutoff. The filtered data were then transformed such that the " $x$ " coordinate axis was defined as the left-right dimension, the " $y$ " coordinate axis was defined as the front-back dimension, and the $(0,0)$ coordinate was positioned at the starting location. Since the movements were constrained to the plane of the table, transformed $z$ coordinates were not analyzed. The computer identified several kinematic events including peak acceleration, peak velocity, and the end of the transport phase of each movement. The end of the transport phase was defined by one of two possible events: the first tangential velocity minimum or the first data frame $<2 \mathrm{~cm} / \mathrm{s}$ after the velocity dropped below $30 \%$ of the peak velocity and stayed below $30 \%$ for at least one more velocity peak (see Spencer and Hund, 2002). After the computer selected the kinematic events on each trial, the directional errors at these locations were computed by calculating the angle between two lines: (1) the line connecting the start location and the spatial position of the finger at the time of the selected kinematic event (e.g., peak velocity), and (2) the line connecting the start location and the target location. Negative directional errors indicate counterclockwise errors relative to the target direction.

For each participant, directional errors were checked for outliers in a two-stage process. In the first stage, the computer identified outlier trials by determining which trials had directional errors larger than the participant's median error \pm 2 standard deviations $(S D s)$ for movements to each target location at each planning time. If the participant had a standard deviation $<5^{\circ}$ for a particular cell of the design (as occurs when, for instance, all movements to a particular target at a particular planning time are equally accurate), the standard deviation was set to $5^{\circ}$ to avoid calling any of these trials "outliers." Conversely, if the participant had a standard deviation $>10^{\circ}$ for a particular cell of the design, the standard deviation was set to $10^{\circ}$ to ensure that at least some of the extreme values in such cells were initially flagged as outliers (see Spencer \& Hund, 2002). Next, all outlier trials were re-analyzed using an interactive version of the automated analysis software to determine if the large errors were due to computer selection mistakes. After this second stage, only one trial was eliminated across all participants.

All of the reported analyses examined directional error at peak velocity. Ghez et al. (1997) examined both peak velocity direction and peak acceleration direction. We focused on the former measure because the timing of peak velocity was more consistent across trials. Note, however, that we obtained a similar pattern of results with peak acceleration direction. Stimulus-response (S-R) intervals were computed from the onset of the target to the onset of movement. Trials were then grouped into $50 \mathrm{~ms} \mathrm{S-R}$ intervals and plotted in histograms. 


\section{Results}

The mean initiation time across all trials was $43.4 \mathrm{~ms}$. Participants' initiation times were significantly faster at the longer planning times, $F(4,1680)=7.07, p<.001$. Average initiation times for all planning times, however, were less than $65 \mathrm{~ms} \mathrm{(30}$ ms: $M=62.4 \mathrm{~ms} ; 80 \mathrm{~ms}: M=56.6 \mathrm{~ms} ; 130 \mathrm{~ms}: M=38.2 \mathrm{~ms} ; 180 \mathrm{~ms}: M=34.9$ $\mathrm{ms} ; 230 \mathrm{~ms}: M=25.0 \mathrm{~ms}$ ). Therefore, participants were able to time their response to the "go" signal with reasonable accuracy.

Movement direction at peak velocity for the $20^{\circ}$ separation condition at different stimulus-response (S-R) intervals is shown in Figure 3. Note that this figure includes data from all participants combined. As can be seen in the figure, at short $\mathrm{S}-\mathrm{R}$ intervals, responses were biased toward $0^{\circ}$. As the $\mathrm{S}-\mathrm{R}$ interval increased, responses moved toward each target, and the distribution of responses became tri-modal by $230 \mathrm{~ms}$. Thus, when the targets were separated by $20^{\circ}$, movement planning appeared continuous. Movement direction at peak velocity for the $80^{\circ}$ separation condition at different S-R intervals is shown in Figure 4. As can be seen in the figure, movements in this condition were tri-modal at all S-R intervals, with relatively few responses in-between the target locations. Although responses at the short planning times were tri-modal, they were not accurate-there were many "guesses" to the incorrect location. This was the case for all S-R intervals shorter than $230 \mathrm{~ms}$. Thus, when the targets were separated by $80^{\circ}$, movement planning appeared discrete.

These results across conditions were also true for individual participants. Data from a representative participant in each condition are shown in Figure 5. Note that we have grouped planning times to increase the number of observations for short $(<130 \mathrm{~ms})$ versus long planning times $(>130 \mathrm{~ms})$. As with the overall data, there was a clear difference across conditions at the short S-R intervals, with a uni-modal response distribution in the $20^{\circ}$ condition and a tri-modal distribution in the $80^{\circ}$ condition.

Next, we examined the time course of movement specification by computing the percentage of observed responses in the correct direction at peak velocity across conditions. In an initial analysis, each response was coded as correct or incorrect based on whether the response was within $\pm 10^{\circ}$ of the target direction. These data are shown in Figure 6a. As can be seen in the figure, when the targets were separated by $20^{\circ}$, the number of responses in the correct direction increased systematically as S-R interval increased. This was also the case at $80^{\circ}$, but the increase occurred at longer S-R intervals and never attained the same degree of accuracy. A chi-square analysis comparing the percent correct in the $20^{\circ}$ and $80^{\circ}$ separation conditions showed a significant difference in accuracy across conditions beginning with the 80-129 ms S-R intervals (all intervals greater than 80-129 ms were also significant, $p<.01$ ). This confirms the faster specification in the $20^{\circ}$ condition evident in Figure 6 a.

We conducted a second analysis of movement specification time using criteria from Ghez et al. (1997). These researchers examined the time course of specification by coding each response as correct or incorrect based on which target it was closest to. Thus, in the $80^{\circ}$ separation, for example, a response could be $39^{\circ}$ from the target location but was still coded as correct, because it was closest to the correct target. Figure $6 \mathrm{~b}$ shows the percentage of correct responses minus the percentage 


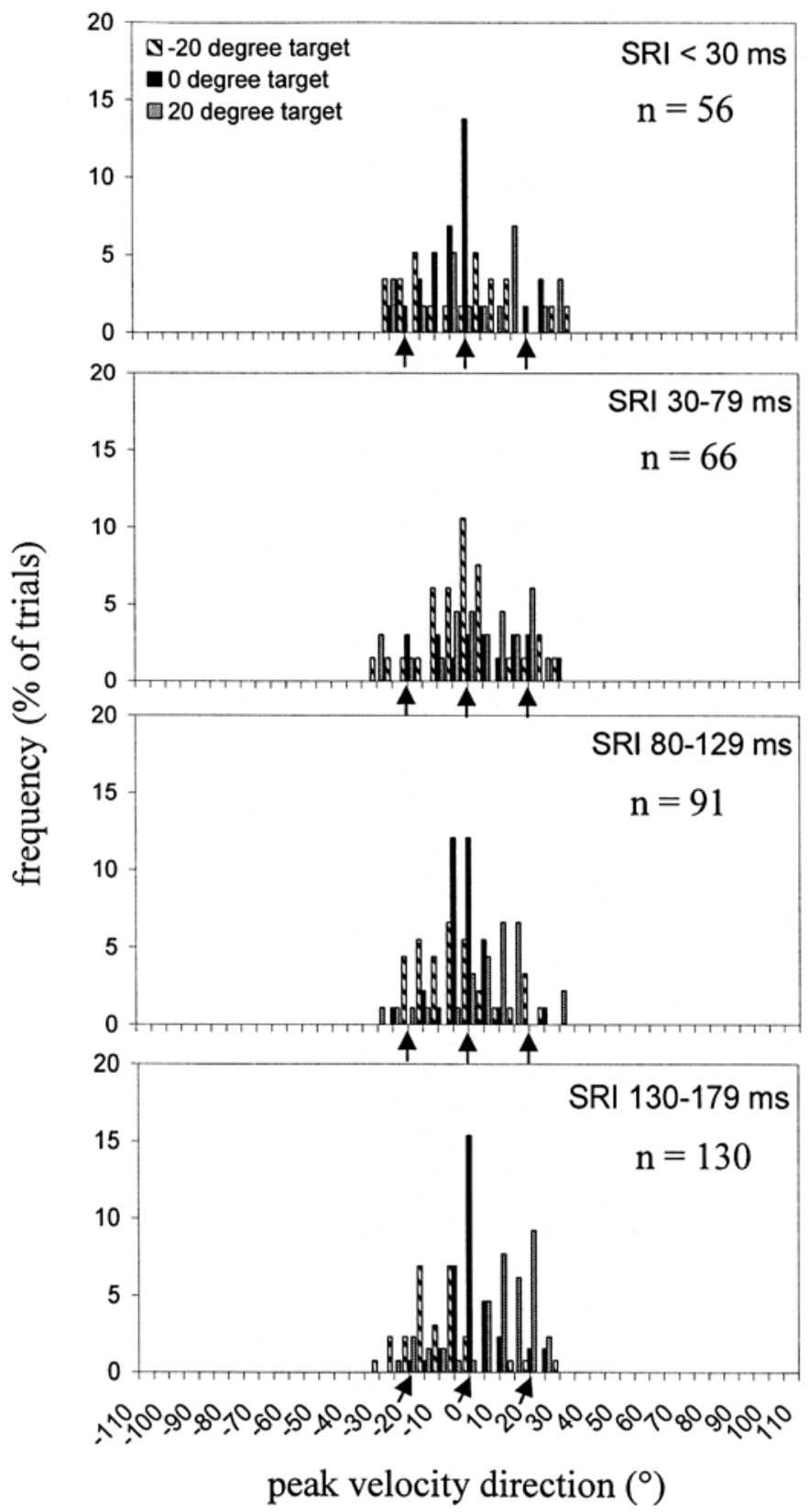

Figure 3-Frequency (percent of trials) of peak velocity direction histograms for Experiment $1,20^{\circ}$ separation, for short to long S-R intervals (SRI) for all seven participants' data combined. Frequency is number of trials to a location for a target divided by total number of trials in S-R interval. Ns are number of trials in each graph. Target locations are marked with arrows. 


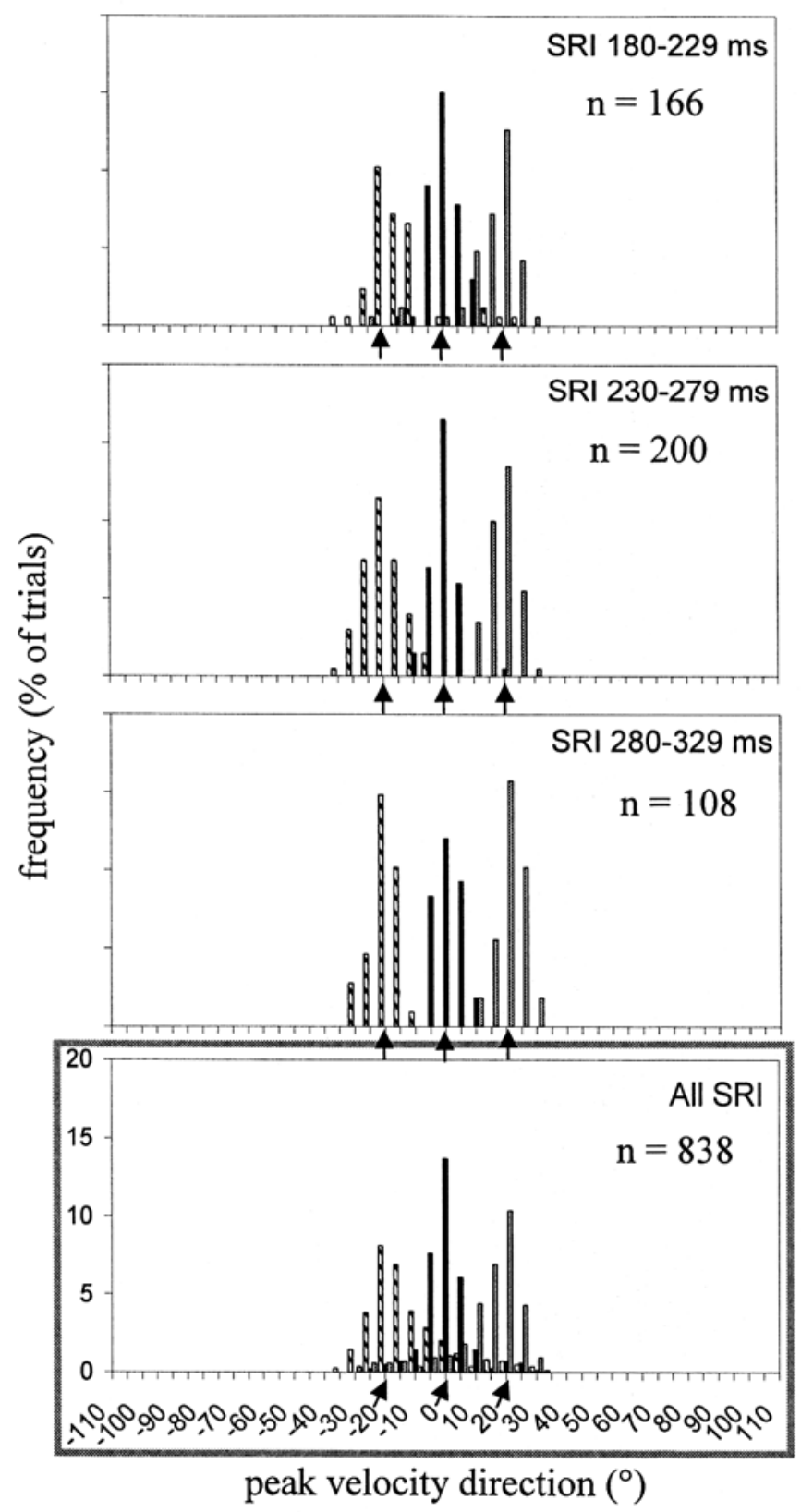

Figure 3, continued 


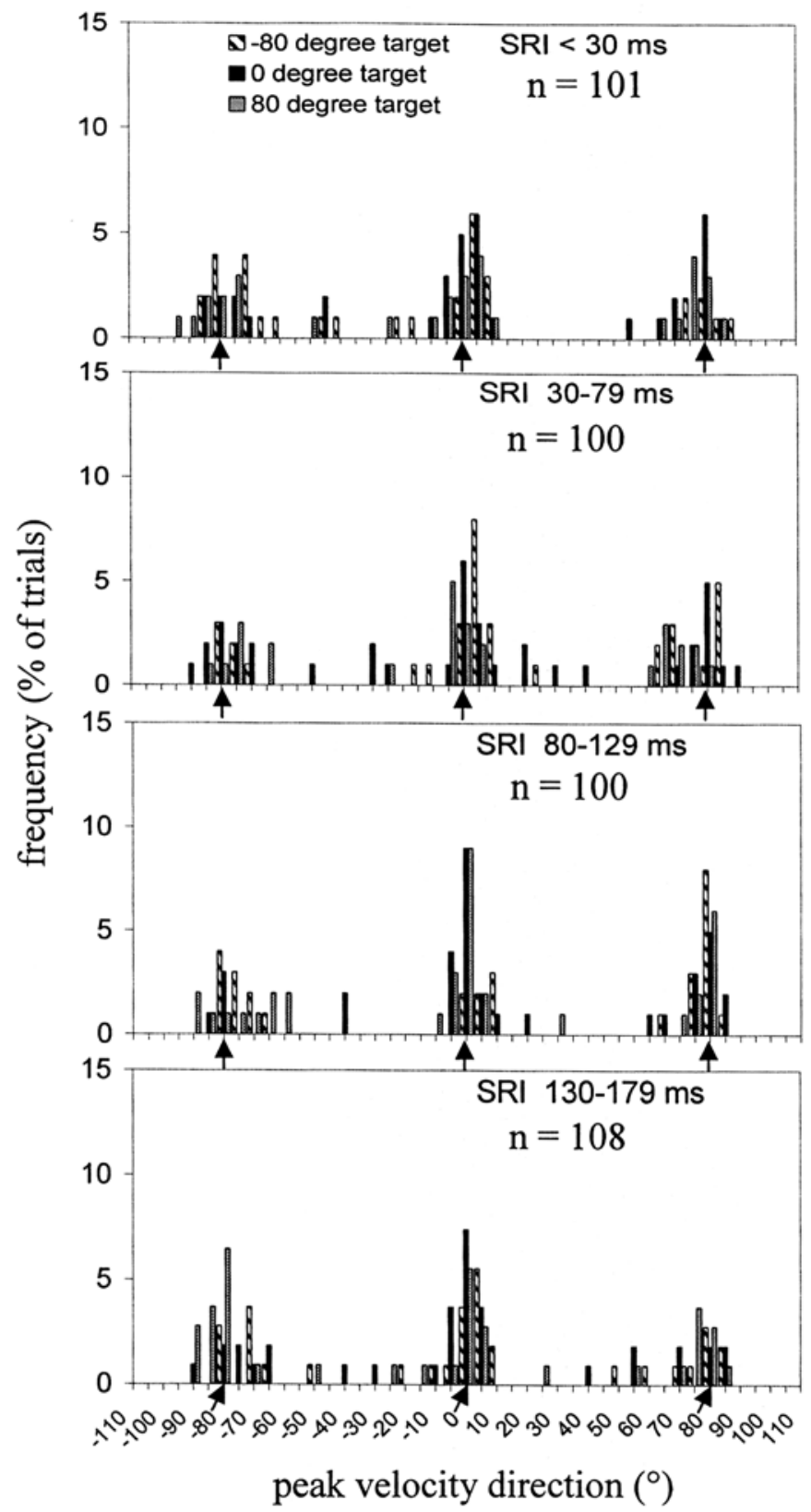

Figure 4-Frequency (percent of trials) of peak velocity direction histograms for Experiment $1,80^{\circ}$ separation, for short to long S-R intervals (SRI) for all seven participants' data combined. Frequency is number of trials to a location for a target divided by total number of trials in S-R interval. Ns are number of trials in each graph. Target locations are marked with arrows. 


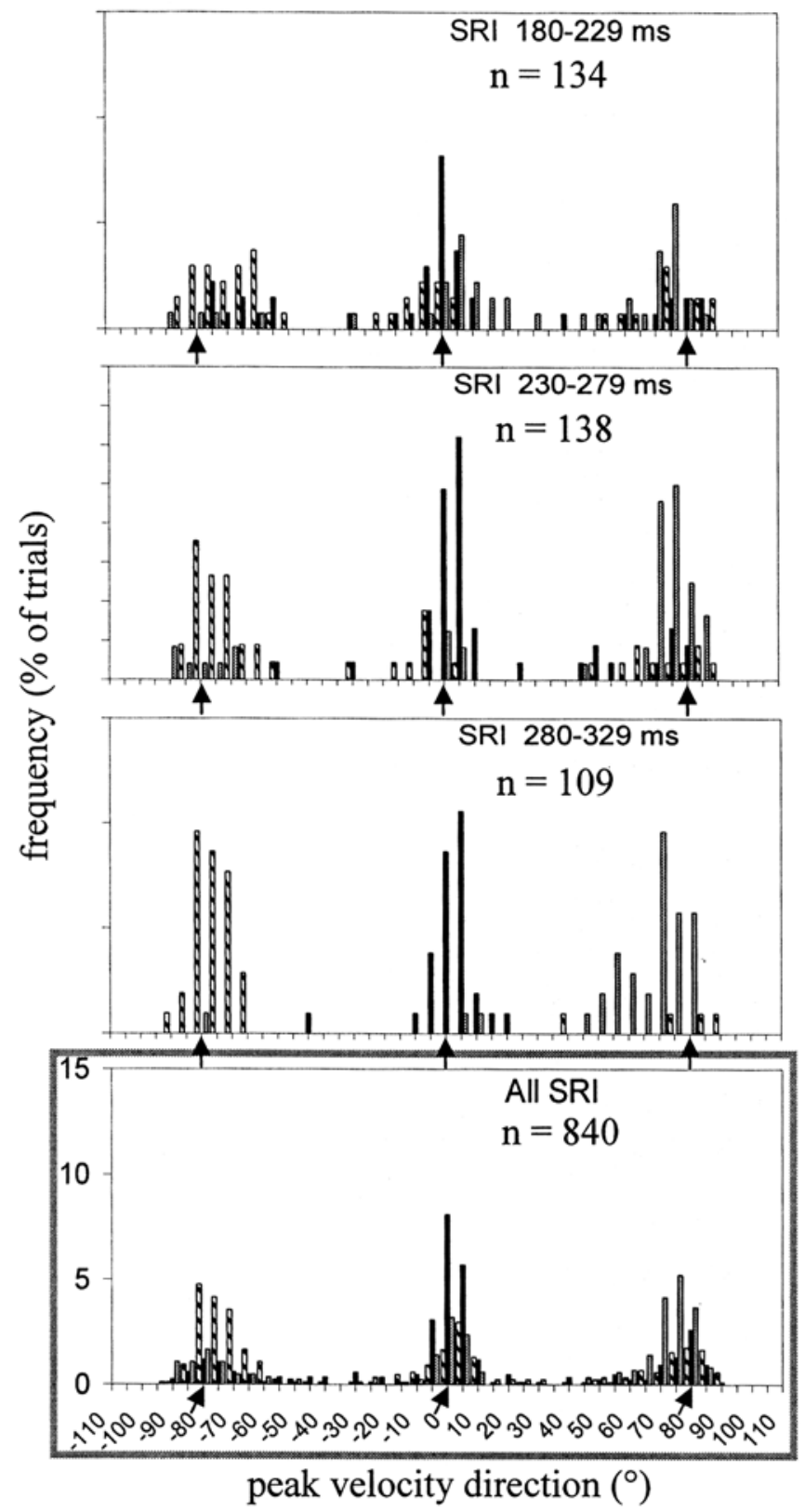

Figure 4, continued 


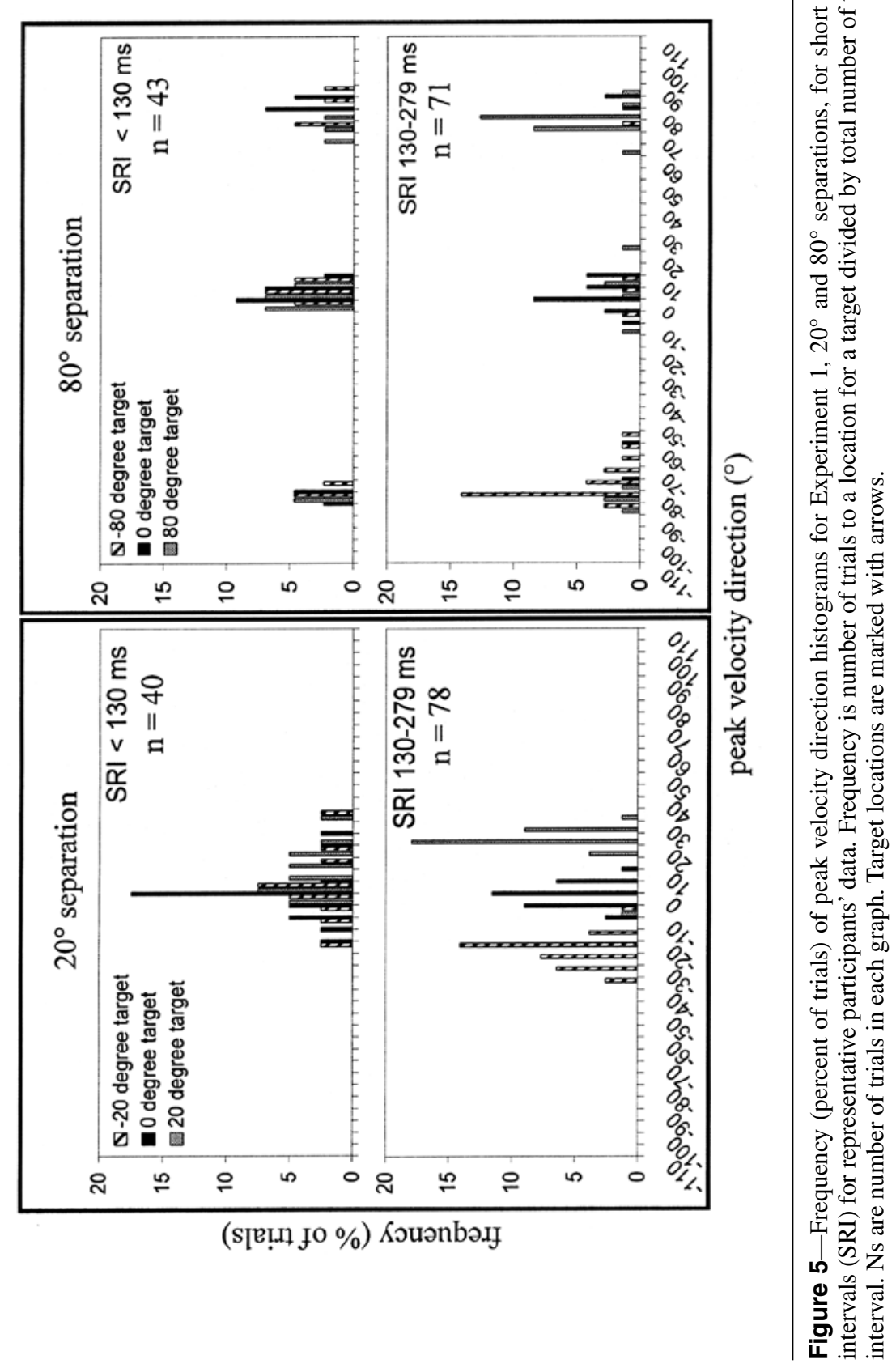




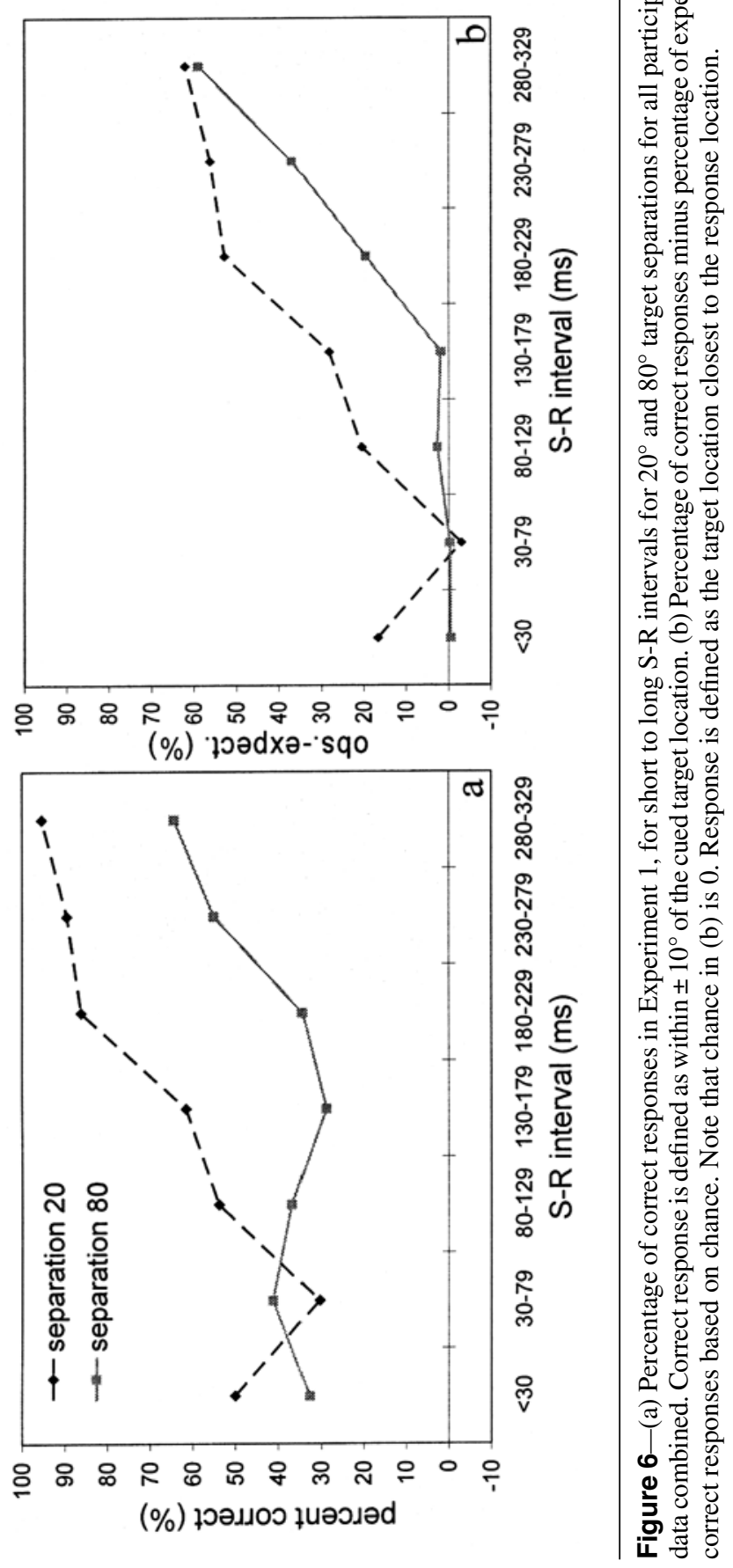


of expected correct responses based on chance (33\%). Note that we have plotted these data in this manner to match figures from Ghez et al. (1997). As can be seen in the figure, results were comparable to the analysis above (see Figure 6a) with two exceptions: specification time was faster for the $80^{\circ}$ condition relative to the previous analysis and performance in the two conditions was comparable at the longest S-R intervals (280-329 ms). This is because the criteria used by Ghez and colleagues were less stringent when applied to the $80^{\circ}$ condition (responses were "correct" within $\pm 40^{\circ}$ of the target versus $\pm 10^{\circ}$ above). A chi-square analysis comparing observed and expected correct responses at each S-R interval for each condition separately found that when the targets were separated by $20^{\circ}$, correct responses significantly exceeded chance at the S-R intervals of 80-129 ms. When the targets were separated by $80^{\circ}$, the number of correct responses significantly exceeded chance at the S-R intervals of 180-229 ms. The faster specification time in the close targets condition replicates results from Ghez and colleagues (1997).

\section{Discussion}

Despite a number of differences in the task, the results of this experiment replicated results from Ghez et al. (1997). Movement planning appeared continuous at the narrow target separation and discrete at the large target separation, and movement specification was faster in the $20^{\circ}$ separation condition (for related results, see Favilla, 2002). This replication demonstrates the robust nature of the original findings by Ghez and colleagues. Of particular note, the continuous and discrete modes were robust to our task context where participants were allowed full view of their hands. Visual inspection of the data indicated that there were very few cases of clear violations of the task instructions, that is, clear corrections: in general, movement direction at peak velocity matched the movement direction at the end of the movement. This is consistent with results from Prablanc and Martin (1992). These researchers used a double-step paradigm to examine differences between open-loop and closed-loop control. They found no significant differences in movement trajectories prior to the endpoint of the movement.

In summary, results from Experiment 1 were consistent with both the two modes account (Ghez et al., 1997) and the DFT (Erlhagen \& Schöner, 2002). Having replicated the main findings in our modified experimental setting, we could test conflicting predictions of the two accounts in Experiment 2.

\section{Experiment 2}

According to the DFT, the two modes proposed by Ghez and colleagues (1997) emerge flexibly from the same underlying system. Importantly, which mode emerges depends on the details of the target, task, and LTM inputs. In our modified version of the task, the sole "preshaping" influence on movement planning is from the LTM field, that is, participants' long-term memory (LTM) of previous responses built-up from trial to trial. In the present study, we attempted to "smear out" participants' LTM by allowing them to make on-line corrections during each trial. We hypothesized that this would create a more distributed activation profile in LTM-even when the targets are far apart—because participants might form multiple movement 
plans on each trial, some of which are likely to occur in between candidate target locations. For example, a participant might plan a movement toward $0^{\circ}$ yet catch a glimpse of the target at $80^{\circ}$ several milliseconds earlier. Guided by the reasonably accurate perceptual memory of the target at $80^{\circ}$, the participant might correct the movement plan on-line, forming a plan centered near $80^{\circ}$.

What might activation in an MP field look like in this case? There are two possibilities for how activation in the MP field might evolve in such corrective situations (for related ideas, see Schöner et al., 1997). Activation in the planning field might "jump" from $0^{\circ}$ to a location near $80^{\circ}$, that is, initial activation at $0^{\circ}$ might be actively suppressed as activation near $80^{\circ}$ is allowed to grow based on the sustained memory of the target (for related effects, see Bastian et al., 1998; Bastian et al., 2003; Erlhagen \& Schöner, 2002). Alternatively, movement plans could rotate continuously from an initial plan at $0^{\circ}$ through intervening target directions, finally settling in on a direction near $80^{\circ}$ (for related ideas regarding spatial drift, see Schutte et al., 2003; Spencer \& Hund, 2002; Spencer \& Schöner, 2003).

Note that these two possibilities specify how motor planning can change in the DFT when corrective movements are allowed, but they do not specify the precise reaching trajectories that would arise from such corrections in planning. To specify this, we would need to implement a more complete motor control model that couples the DFT to a system capable of generating realistic trajectories. There are several models that move in this direction (e.g., Dounskaia, Swinnen, \& Walter, 2000; Feldman \& Levin, 1995; Schöner et al., 1997), but such detailed analyses of the coupling between motor planning and reach trajectories are beyond the scope of the present study (for a discussion of the issues involved in the challenge, see Schöner, 1995). Rather, the goal here is to specify how we think corrective motor plans can be achieved/implemented in the DFT, how such corrections lead to the predictions tested in the present experiment, and what core assumptions we make with the particular implementation we selected.

The simulations in Figure 7 show the model's behavior and resultant LTM fields that emerged from 12 simulations of the DFT without (Figure 7a) vs. with (Figure $7 d)$ corrections allowed. The targets in these simulations were far apart $\left(80^{\circ}\right.$ separation) and were presented for $30 \mathrm{~ms}$. Note that the task input was removed in both simulations, consistent with a key modification to the task space probed in Experiment 1. Also, the simulation with corrections was allowed to run longer on each trial to allow time for the model to update the movement plan. Specifically, the total trial length without corrections was $780 \mathrm{~ms}$, and the trial length with corrections was $2,530 \mathrm{~ms}$. All other simulation details were identical to the simulations presented earlier.

To implement corrections in the MP field, we made one change to the target input - it was sustained at a lower activation level in PI after the target disappeared from the task space (see top layer in Figure 7d). This reflects participants' use of a sustained perceptual memory of the target to update motor plans as the movement unfolds during a trial. What is the effect of the sustained target input? Figure 7e shows a time slice from just after the model developed a peak in the MP field. As can be seen in the figure, the model formed an initially incorrect plan to move to a direction in between the left and center targets - the model made an initially continuous-looking response even though the targets were separated by $>60^{\circ}$. Importantly, after the short target presentation, a perceptual trace of the target was 

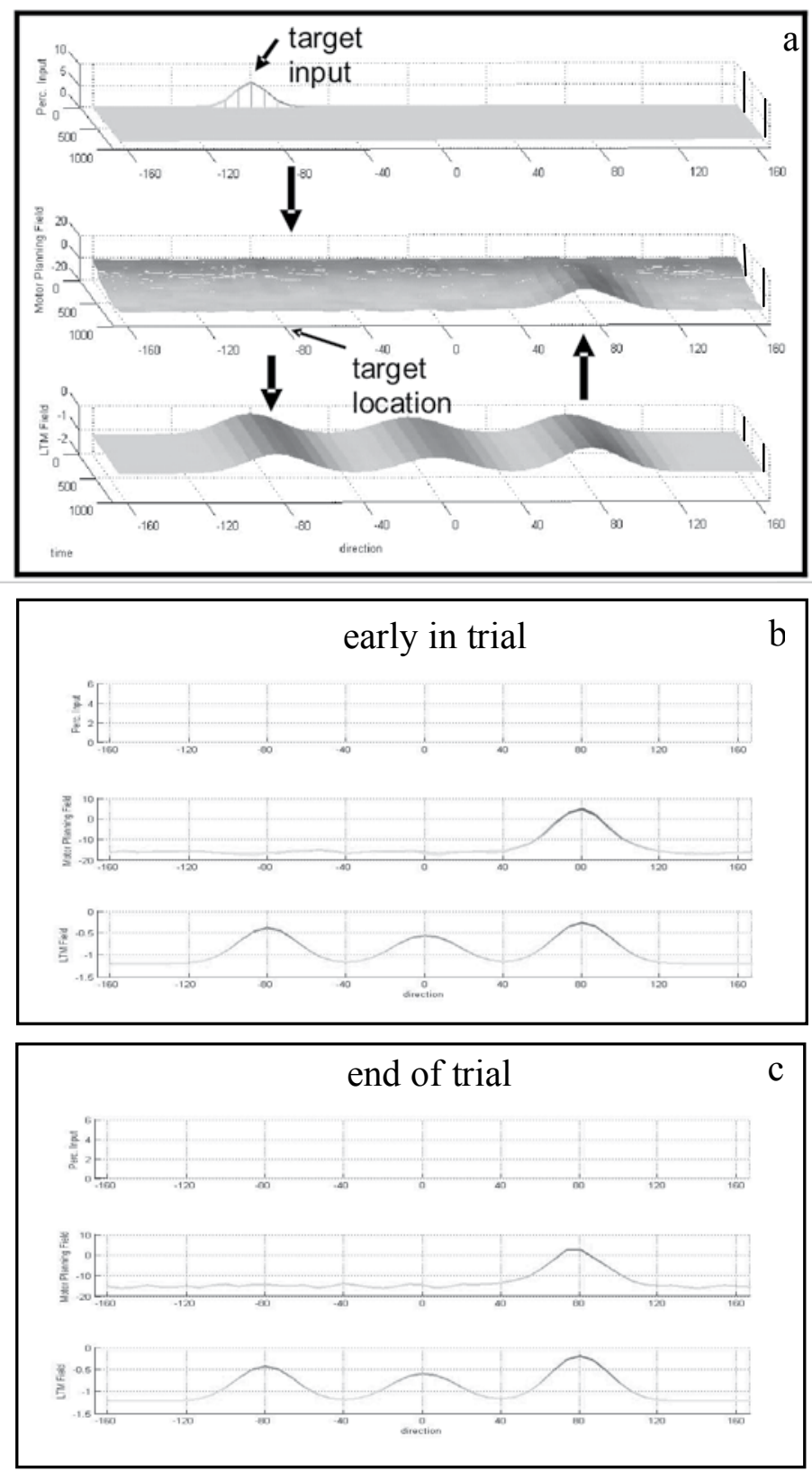

Figure 7-Simulations of the DFT with a large target separation without (panels a-c) and with (panels d-f) corrections. Location is on the $x$-axis, activation is on the $y$-axis, and time is on the $z$-axis (in panels a and d). The top layer in each panel is perceptual input, the center layer is the motor planning field, and the lower layer is the longer-term memory field. See text for additional details. 

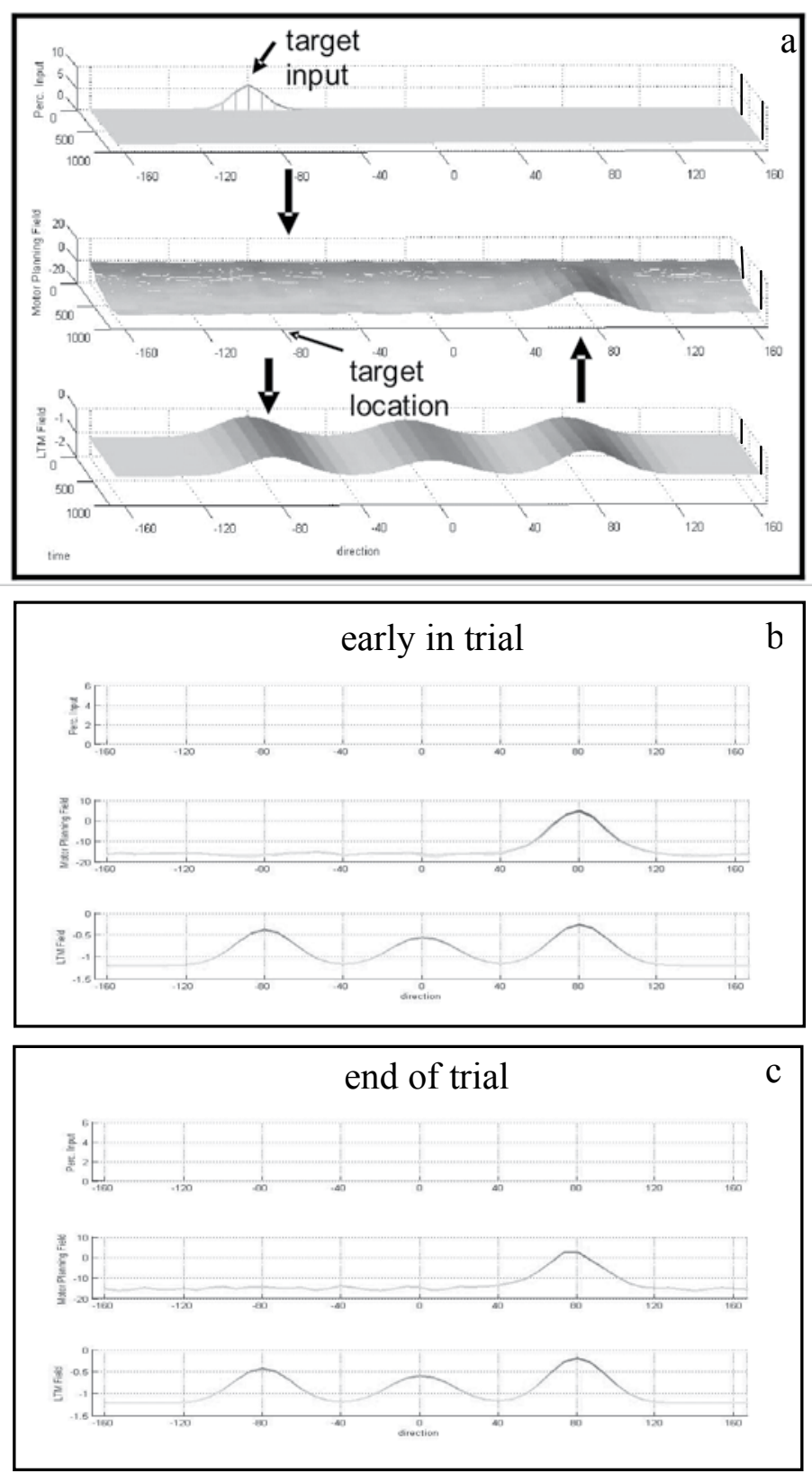

Figure 7, continued 
maintained. This caused the peak of activation in the MP field to "drift" or rotate toward the sustained target input (see circled activation trace in the center layer of Figure 7d), such that the model formed a more accurate movement plan by the end of the simulation (see central layer of Figure 7f). This would, in turn, lead to on-line corrections in the reach trajectory, the details of which we did not model. Importantly, the rotating motor plan in Figure 7d leaves a "smeared out" trace of activation in the LTM field (see bottom layer of Figure 7d) which creates a graded activation profile in this field with high levels of activation in between the left and center target locations (see bottom layer of Figure 7f). This, in turn, makes the model more likely to show continuous-looking initial responses on subsequent trials, even in this condition where the targets were far apart. Note that many complex activation profiles are possible in the LTM field when corrections are allowed. The details of which pattern emerges depends on the trial-to-trial target sequence, the planning time on each trial, and the noise level.

The fact that many complex patterns can emerge in the LTM field raises the issue of how strong the predictions tested in this experiment really are. More generally, the simulation results in Figure 7 raise the question of how strongly our predictions rest on the details of the correction mechanism we proposed and, similarly, what we must assume for this mechanism to work. We address these questions in turn below.

Although the LTM mechanism in the DFT can lead to many complex patterns when corrections are allowed, in a timed initiation paradigm with short planning times and reasonably close targets, these complex patterns will all have (at least) one feature in common - traces of activation in between the actual target directions. Importantly, this common feature will reliably lead to the predicted effect tested herein. Thus, while it is possible that discrete-like performance could occur when all targets are, for instance, $120^{\circ}$ apart and corrections are allowed, we suspect that even in such extreme cases, continuous-looking performance is still likely. This is because even a few accidental responses in between the actual target locations can lead to "snowballing" influences in the DFT. In particular, whether an accidental response was initially caused by a rotating motor plan or a "jumping" plan (see discussion above), the LTM trace left by each "accident" can preshape similar responses in between the targets, particularly on subsequent short planning trials when participants must effectively guess where the target is. As such traces accumulate, motor plans are more likely to "drift" or rotate, which further "smears out" LTM traces, leading to a greater tendency for continuous-looking response distributions. In this context, we note that neural and behavioral evidence shows that rotational effects occur across a relatively large span of movement directions. Specifically, work examining both reaction times in humans and neuronal population dynamics in non-human primates suggests that movement plans can rotate continuously for target separations of at least $90^{\circ}$ (Georgopoulos et al., 1989), and potentially as large as $140^{\circ}$ (Georgopoulos \& Massey, 1987).

The second question raised by the simulation results in Figure 7 is how strongly our prediction rests on the details of the correction mechanism shown in this simulation. The central assumption we made is that there is some sustained perceptual memory of the target direction when participants show a corrective movement. Beyond this assumption, the details of how motor plans rotate and/or "jump" to form new motor plans in the DFT are basic characteristics of the dynamics inherent in the 
MP field. This means that we are making relatively modest additional assumptions about how corrective plans come about in the present study (i.e., through some sustained memory of the target location). It also means that the predictions we test here are tightly connected to the original model proposed by Erlhagen and Schöner (2002) because they result from the same mechanisms in the model that produce the core features of performance in the standard timed-initiation paradigm (e.g., the influence of preshaping inputs on planning, the time-dependent dynamics that form and maintain activation peaks in the MP field, and so on).

To summarize, the DFT predicts that allowing corrections in the absence of task input should lead to a more distributed pattern of activation in LTM when targets are far apart $\left(>60^{\circ}\right)$, with traces in between the actual target directions. This, in turn, should produce movement plans to targets in between the actual targets at short planning times in the timed initiation paradigm. Thus, response distributions should look "continuous" even when the targets are far apart. The two modes account, by contrast, predicts that responses should look discrete because the target separation is greater than $60^{\circ}$.

\section{Method}

\section{Participants}

A total of 12 adults ( $M=23.0$ years; $S D=4.38$ years $)$ participated in this study. Participants were recruited from the undergraduate and graduate student bodies at the University of Iowa and at Indiana University. Participants received either course credit or payment for their participation. All participants were right-handed. The number of females and males was roughly balanced across conditions. Data from eight participants were collected at Indiana University. Data from one participant were not included in the analyses, because the participant did not come for a second session. This study was approved by the ethics committees of Indiana University and the University of Iowa, and it was performed in accordance with the ethical standards laid down in the 1964 Declaration of Helsinki. All participants gave their informed consent before participating in the study.

\section{Materials}

Materials were identical to those used in Experiment 1, except for slight hardware differences between the table at Indiana University and the University of Iowa (see Spencer \& Hund, 2002). From the viewpoint of the participant, the table at Indiana University appeared the same as the table at the University of Iowa.

\section{Task, Procedure, and Feedback}

The task, procedure, and feedback were identical to the task, procedure, and feedback used in Experiment 1 with one exception: participants were instructed that they could make corrections after the initiation of the movement to find the exact spot where they thought the spaceship was hiding. 


\section{Experimental Design and Method of Analysis}

As in Experiment 1, participants were randomly assigned to one of the two experimental conditions where targets were either separated by $20^{\circ}$ or $80^{\circ}$. The method of analysis was identical to Experiment 1 with the exception that we computed three additional kinematic measures: (1) peak curvature of the movement trajectory during the central portion of the transport phase (i.e., between peak acceleration and $80 \mathrm{~ms}$ prior to the end of the transport phase); (2) maximum deviation of the trajectory from a straight-line path during the central portion of the transport phase; and (3) the straightness of the trajectory defined as the ratio between the virtual path (a straight line from the starting to ending point) and the actual hand path length (values closer to 1.0 indicate straighter paths; see Thelen et al., 1996). As a result of the two-stage examination of outliers (see Method of Analysis section in Experiment 1 ), a mean percentage of $0.51 \%$ of trials were eliminated across participants due to large errors ( 8 of 12 participants had no outliers removed and the maximum percentage of trials removed for any one participant was $4.58 \%$ ).

\section{Results}

The mean initiation time across all trials was $57.1 \mathrm{~ms}$. As in Experiment 1, initiation times were significantly faster at the longer planning times, $F(4,1538)=11.27, p$ $<.001$. Average initiation times for all planning times, however, were less than 80 ms (30 ms: $M=76.7 \mathrm{~ms} ; 80 \mathrm{~ms}: M=66.5 \mathrm{~ms} ; 130 \mathrm{~ms}: M=60.8 \mathrm{~ms} ; 180 \mathrm{~ms}: M=$ $43.8 \mathrm{~ms} ; 230 \mathrm{~ms}: M=37.8 \mathrm{~ms}$ ). Thus, once again, participants were able to time their responses to the "go" signal with reasonable accuracy.

Movement direction at peak velocity for short to long S-R intervals for the $20^{\circ}$ separation condition is shown in Figure 8. Note that the figure includes data from all participants in the $20^{\circ}$ separation condition. As in Experiment 1, responses in the $20^{\circ}$ separation condition were uni-modal at the short S-R intervals. Thus, as expected, allowing participants to correct their movements did not affect the planning mode for close targets. Rather, planning remained continuous.

Results for the $80^{\circ}$ condition are shown in Figure 9. As can be seen in the figure, movement planning in this condition was more continuous relative to Experiment 1. Participants' movement directions at the shortest S-R intervals $(<30$ ms) spanned the target range with many responses in between the target locations and poor correspondence with the actual target presented. For instance, responses to the $80^{\circ}$ target were distributed throughout the range from $-80^{\circ}$ to $+80^{\circ}$. The tendency to respond in between the target locations remained at the longer S-R intervals, but the response distributions for trials to each target gradually became more consolidated and centered over the correct target. Notably, however, there were still several responses in between the target locations at the longest S-R interval. This mirrors the relatively slow specification time observed in the $80^{\circ}$ condition from Experiment 1.

As in Experiment 1, the pattern of results across conditions was also evident in the performance of individual participants. Data from a representative participant in each condition are shown in Figure 10. As before, responses were grouped across short and long planning times. As in the overall data, responses at the short planning times looked continuous in both conditions, with responses spanning the 


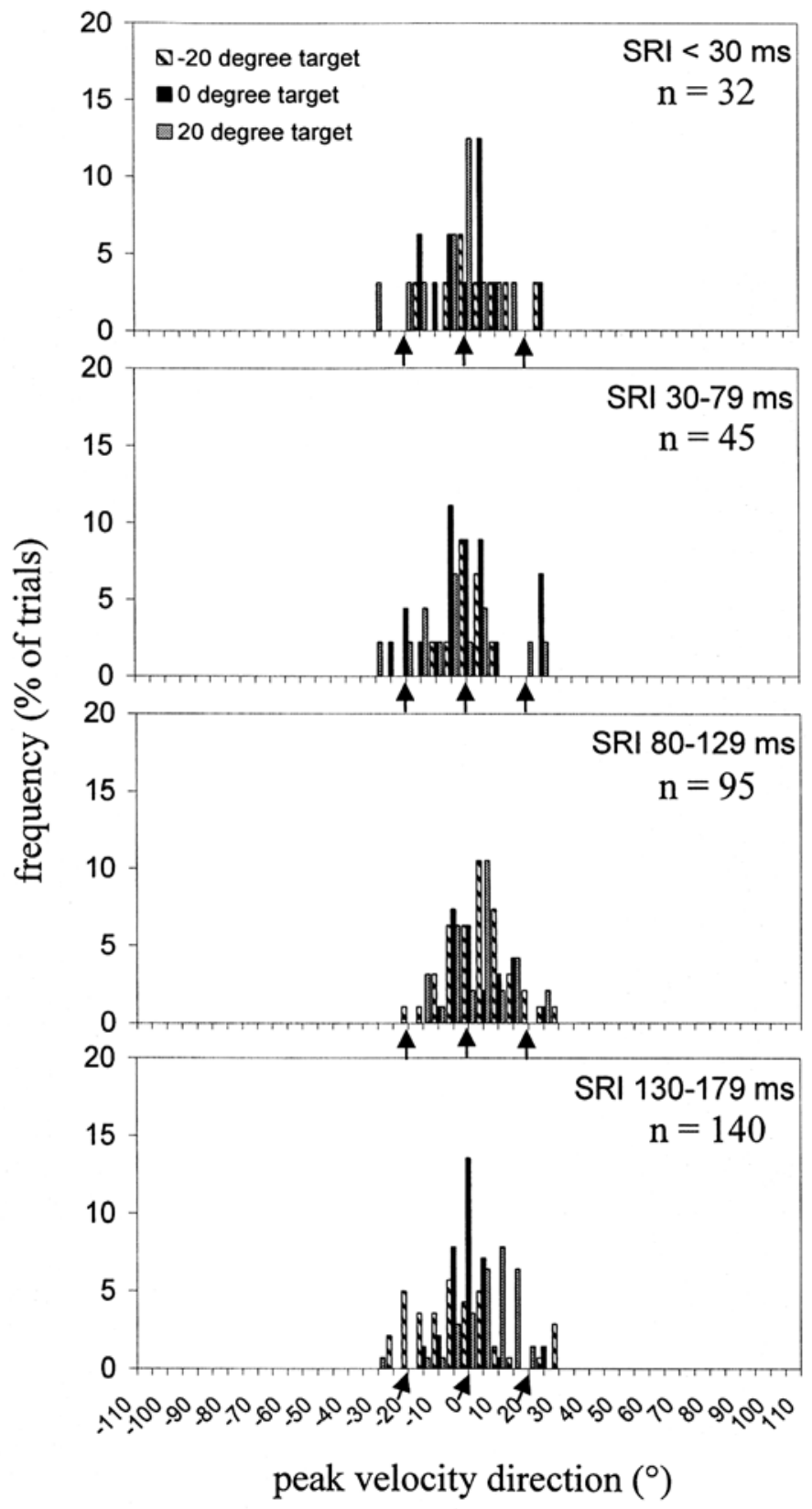

Figure 8-Frequency (percent of trials) of peak velocity direction histograms for Experiment $2,20^{\circ}$ separation, for short to long S-R intervals (SRI) for all six participants' data combined. Frequency is number of trials to a location for a target divided by total number of trials in S-R interval. Ns are number of trials in each graph. Arrows mark the target locations. 


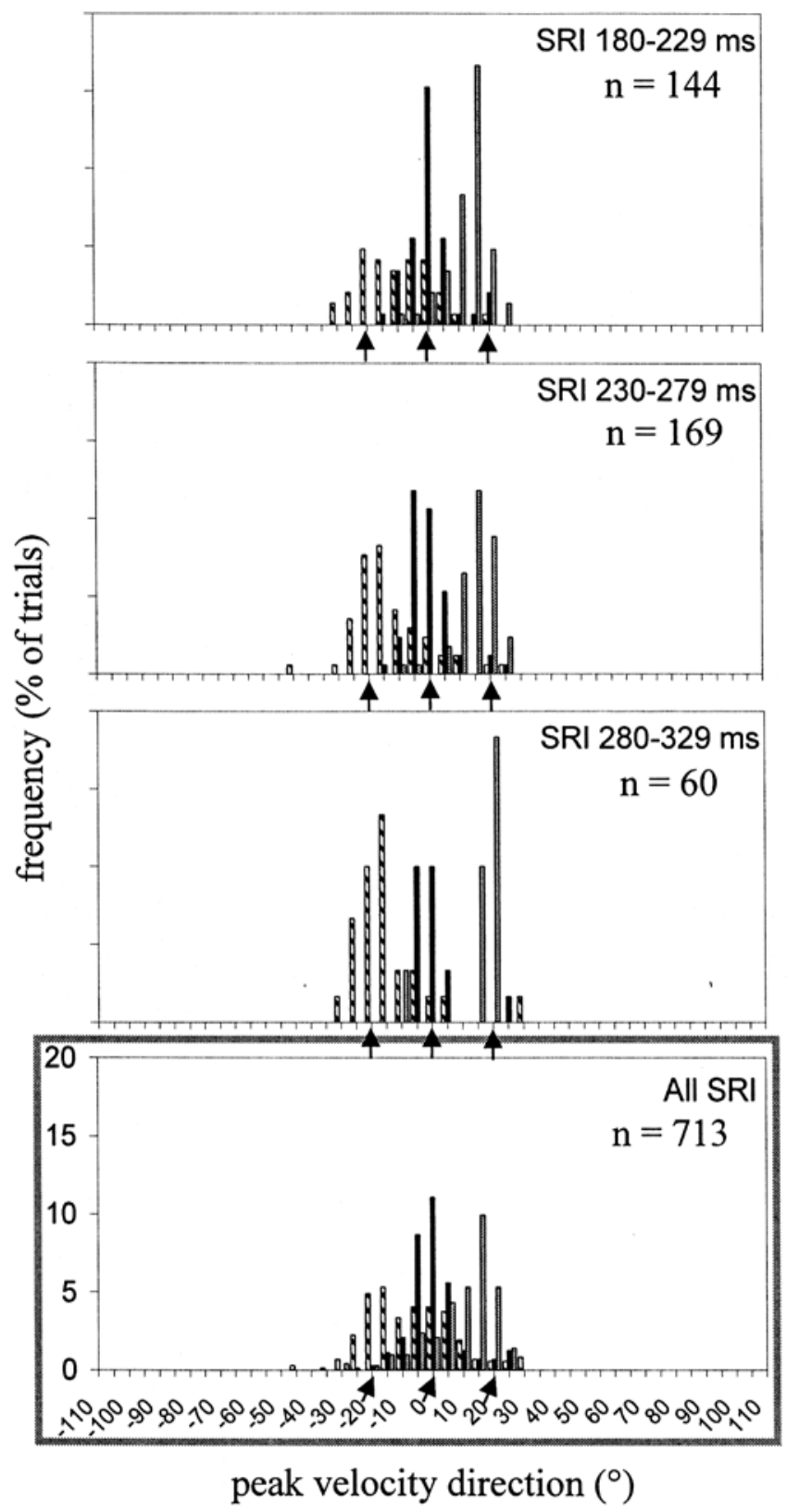

Figure 8, continued 


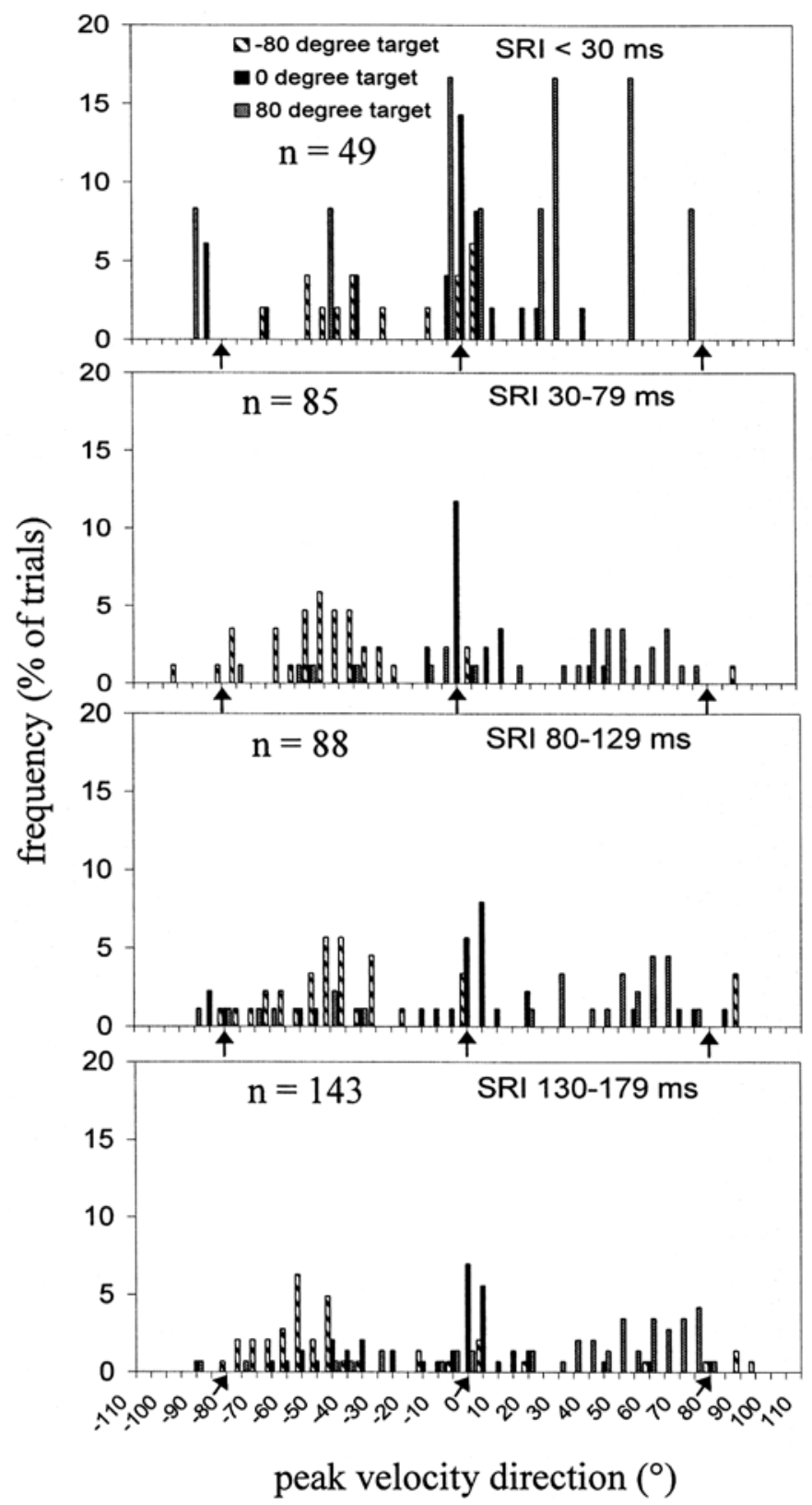

Figure 9-Frequency (percent of trials) of peak velocity direction histograms for Experiment $2,80^{\circ}$ separation, for short to long S-R intervals (SRI) for all six participants' data combined. Frequency is number of trials to a location for a target divided by total number of trials in S-R interval. Ns are number of trials in each graph. Target locations are marked with arrows. 


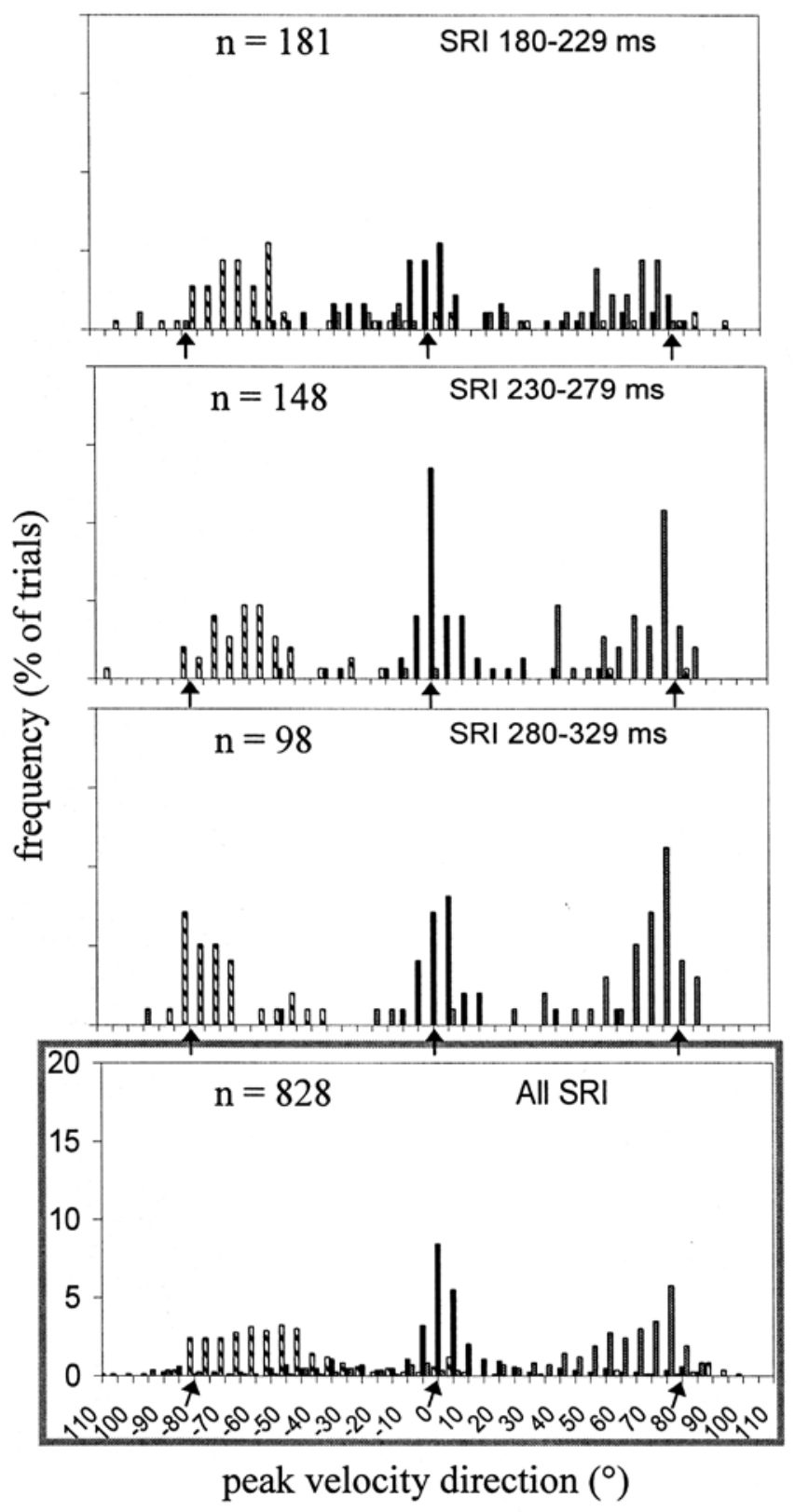

Figure 9, continued 


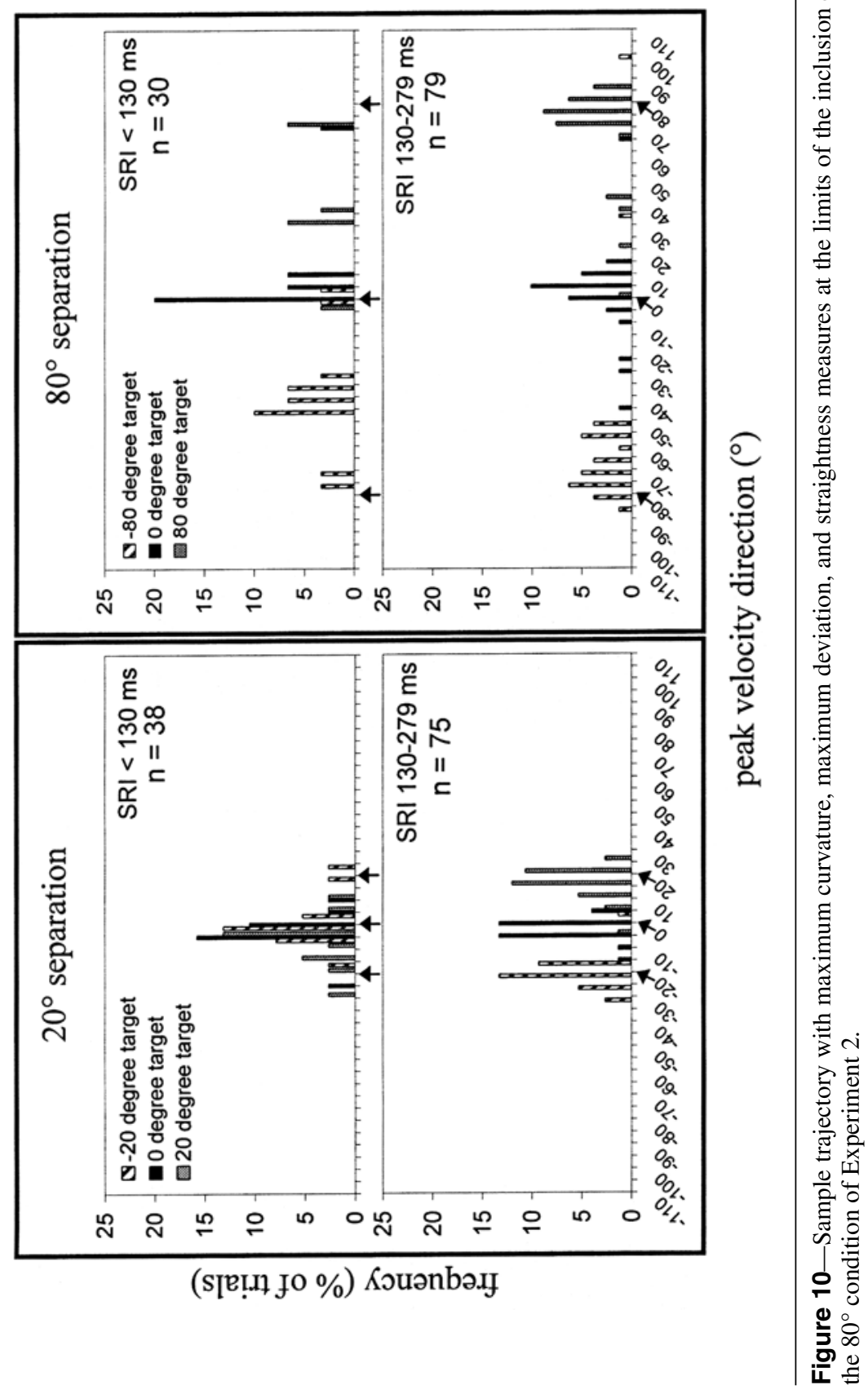


range of the target set. Critically, data from the $80^{\circ}$ condition contrast sharply with the performance of the individual participant in the $80^{\circ}$ condition of Experiment 1 (see Figure 5).

Although data from the $80^{\circ}$ condition suggest that participants used the continuous planning mode, it is possible that responses in between the targets reflect early corrections rather than initial movement plans to directions in between the target locations. That is, in some cases, peak velocity might have occurred after the beginning of a movement correction. In these cases, an initially "discrete" response might be masked by a corrective movement in between the targets. To determine whether this was the case, we examined the timing of peak velocity relative to the straightness and curvature of the trajectories. Movements with peak velocities that occurred within $5 \mathrm{~ms}$ of or following peak curvature were removed from analysis provided that the movement trajectory deviated substantially from a straight line path. To be considered linear and remain in the analysis, the straightness measure had to be greater than or equal to 0.99 , the maximum curvature had to be less than or equal to $0.15 / \mathrm{cm}$, and the maximum deviation from a straight-line trajectory had to be less than or equal to $0.67 \mathrm{~cm}$.

A sample trajectory at the limits of all three criteria is depicted in Figure 11. As can be seen in this figure, the criteria used were very conservative because we wanted to make sure that evidence of the continuous mode of motor planning in the

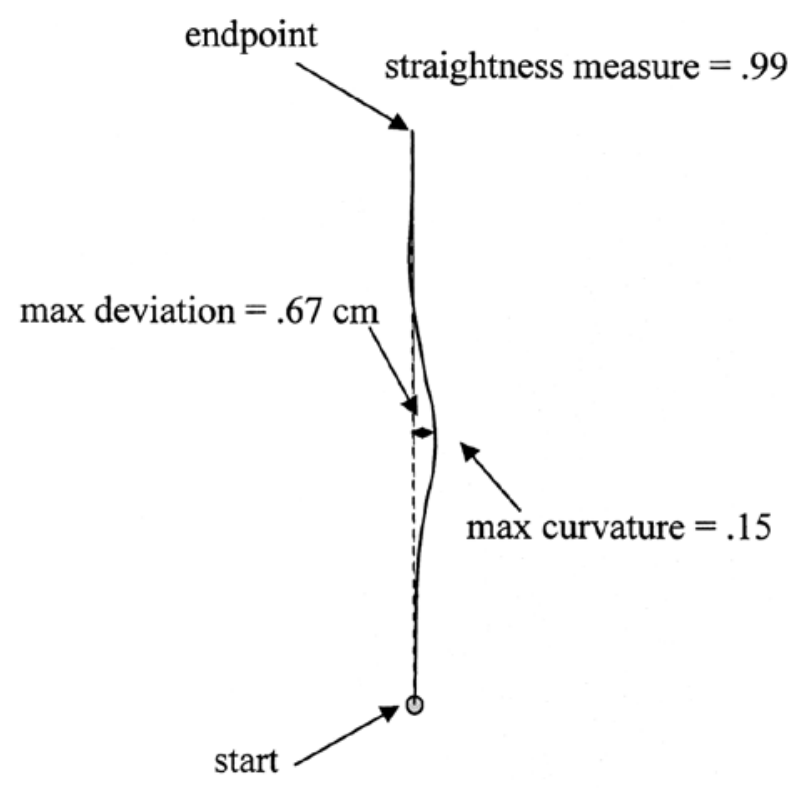

Figure 11-Frequency (percent of trials) of peak velocity direction histograms for Experiment $2,80^{\circ}$ separation, for short to long S-R intervals (SRI) following the removal of movements that were not linear and had peak velocity occur after peak curvature. Frequency is number of trials to a location for a target divided by total number of trials in S-R interval. Ns are number of trials in each graph. Target locations are marked with arrows. 
$80^{\circ}$ condition reflected participants' initial movement plans. Use of these criteria resulted in the removal of $43 \%$ of trials. Although this is a large percentage, the conservative criteria ensured that our follow-up analyses would not be influenced by early corrective movements. To determine whether the included trials were more or less accurate than the excluded trials, such that this resulted in more correct responses, we examined mean error at peak velocity. Mean error to the $0^{\circ}$ target and $-80^{\circ}$ target was slightly lower for the included trials than the excluded trials; however, mean error to the $80^{\circ}$ target was slightly higher for the included trials than the excluded trials. (Included trials: $80^{\circ}$ target $M=-31.90, S D=39.5 ; 0^{\circ}$ target $M=3.81, S D=32.59 ;-80^{\circ}$ target $M=40.09, S D=52.96$; Removed trials: $80^{\circ}$ target $M=-44.63, S D=46.58 ; 0^{\circ}$ target $M=-14.41, S D=26.67 ;-80^{\circ}$ target $M=27.10, S D=28.18$ ).

Histograms of peak velocity direction for all included trials for the $80^{\circ}$ condition are shown in Figure 12. As can be seen in the figure, movement planning in this condition still showed evidence of the continuous planning mode. Participants showed a tendency to move toward the center target at short planning times, and, critically, there were many responses in between the target locations. As the S-R intervals increased, the response distributions gradually moved toward the correct locations.

To compare performance in the present experiment to Experiment 1 more directly, we computed difference histograms across the $80^{\circ}$ separation conditions of Experiments 1 and 2 for short and long S-R intervals (Figure 13). Specifically, for each movement direction, we subtracted the percentage of movements in that direction in Experiment 1 from the percentage of movements in that direction in Experiment 2 (positive values indicate more movements in a particular direction in Experiment 2). If motor planning is more continuous in the present experiment, there should be more movements in between the target directions in Experiment 2, and more movements at the actual target directions in Experiment 1. As can be seen in the figure, this was the case. The positive regions in this figure (light grey shading) are all centered in between the targets, while the negative regions in this figure (black stripes) are centered at the targets.

In a final set of analyses, we examined the time course of movement specification using the two criteria from Experiment 1. In a first analysis, responses at peak velocity were considered correct when they were within $\pm 10^{\circ}$ of the target direction. These data are shown in Figure 14a (results from Experiment 1 are shown for comparison). As can be seen in the figure, movement specification was once again faster in the $20^{\circ}$ than the $80^{\circ}$ condition. A chi-square analysis showed a significant difference in accuracy across conditions beginning with the 130-179 ms S-R intervals (all intervals greater than 130-179 ms were also significant, $p<$ $.01)$. Note that the difference in the rate of movement specification across conditions became significant at shorter planning times in Experiment 1 (80-129 ms). As can be seen in Figure 14a, this difference between experiments was caused by slower specification in the $20^{\circ}$ condition of the present experiment. This was confirmed by a direct comparison of the data from the $20^{\circ}$ conditions across experiments. A chi-square analysis revealed that the percent correct in Experiment 1 was significantly greater than in the present experiment at the following intervals: $80-129$ $\mathrm{ms}$ and all intervals $180-229 \mathrm{~ms}$ or greater $(p<.05)$. Note that there were no significant differences in the rate of specification across the $80^{\circ}$ conditions from the two experiments. 


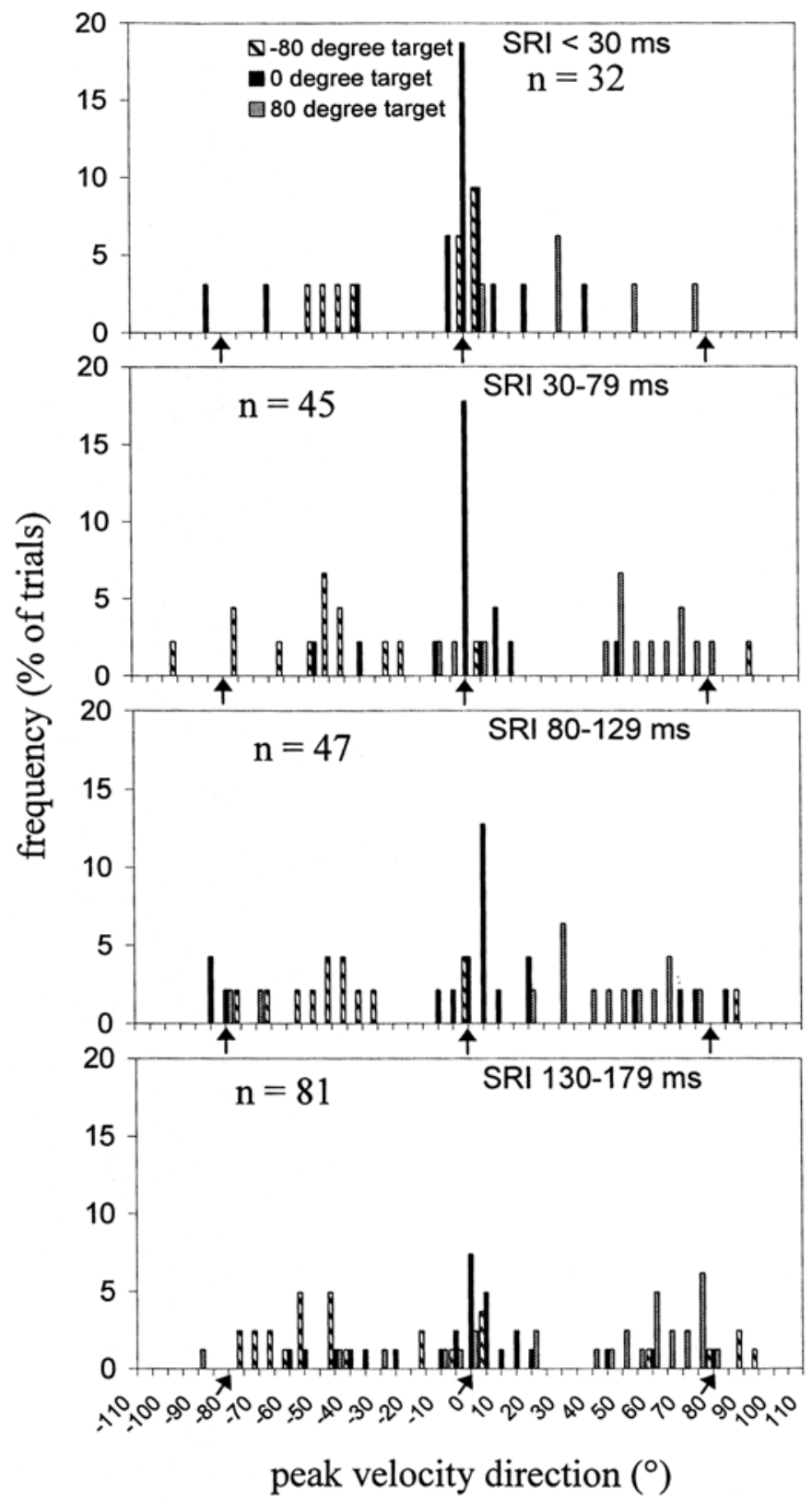

Figure 12-Frequency (percent of trials) of peak velocity direction histograms for Experiment $1,20^{\circ}$ and $80^{\circ}$ separations, for short to long S-R intervals (SRI) for representative participants' data. Frequency is number of trials to a location for a target divided by total number of trials in S-R interval. Ns are number of trials in each graph. Target locations are marked with arrows. 


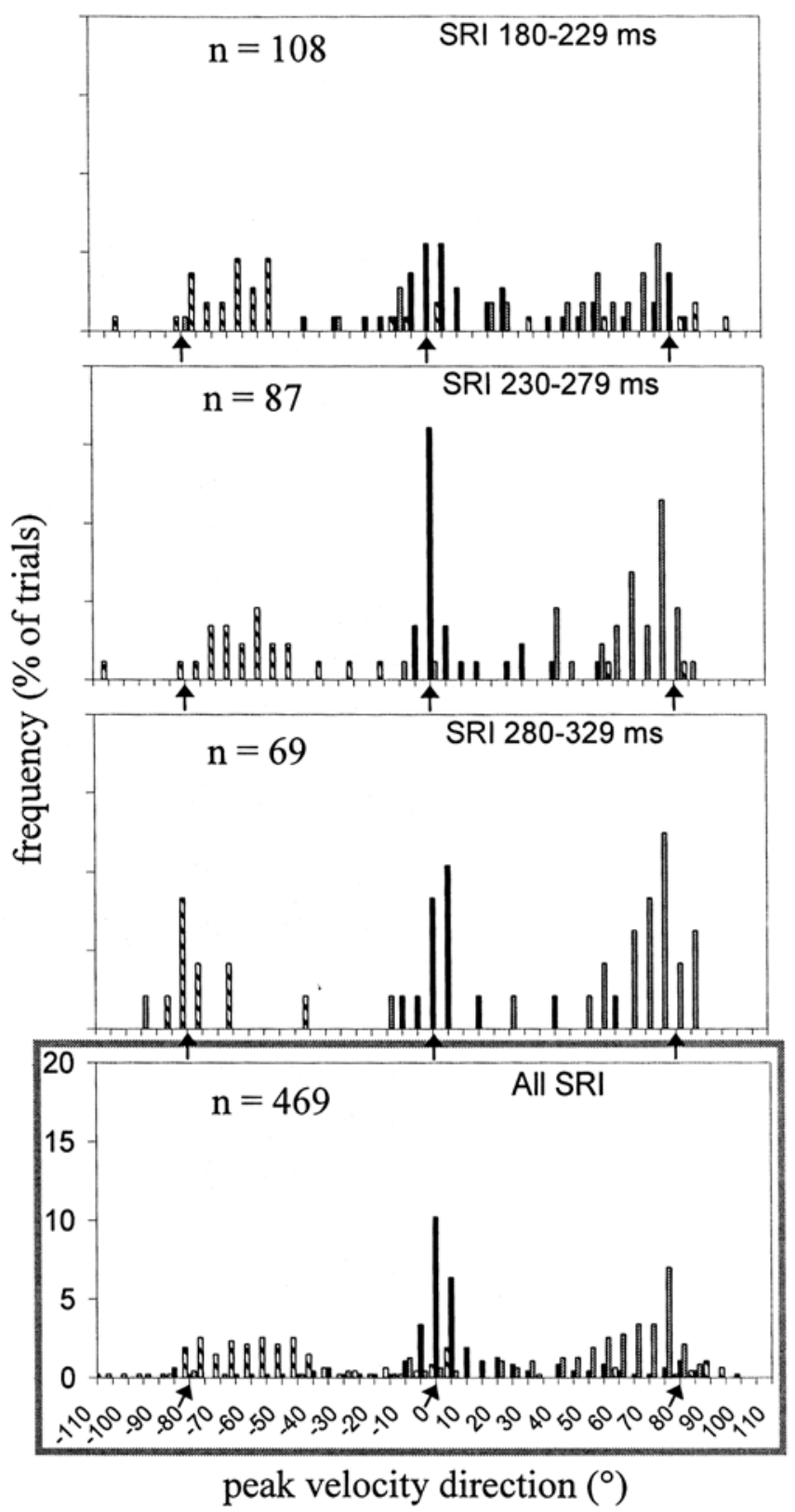

Figure 12, continued 


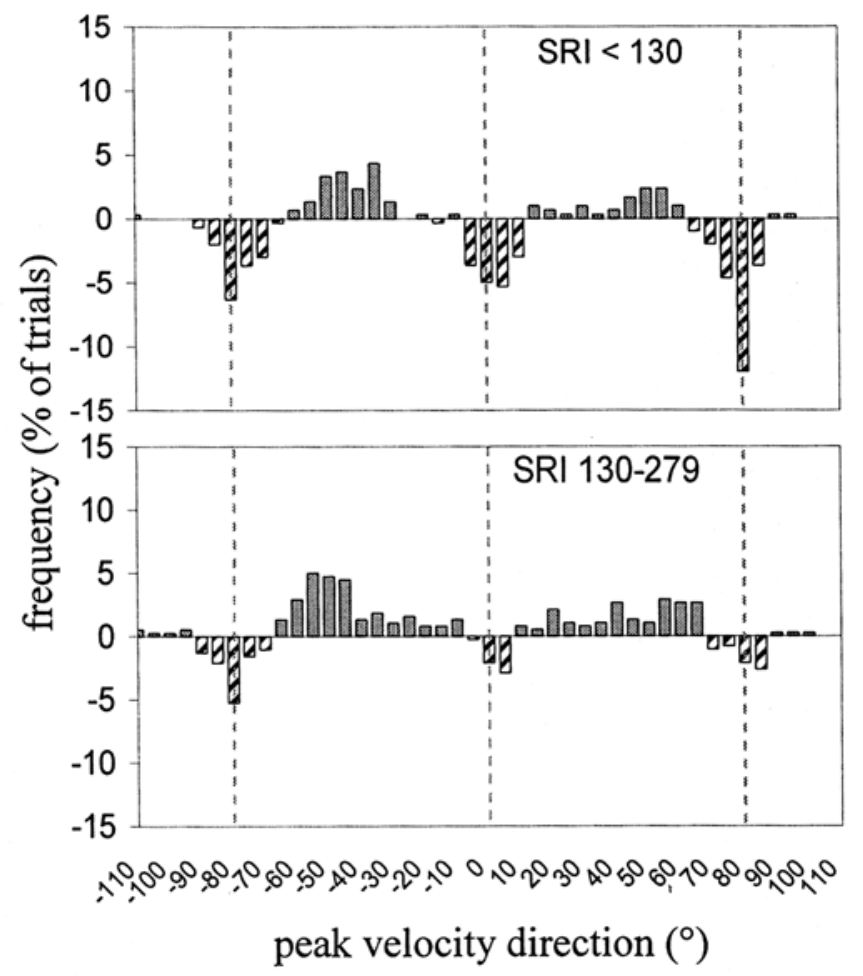

Figure 13-Frequency (percent of trials) of peak velocity difference histograms for $80^{\circ}$ separation. Positive values are more movements in that direction in Experiment 2. Negative values are more movements in that direction in Experiment 1.

Figure 14b shows the time course of movement specification across conditions using the criteria from Ghez et al. (1997). As in the analysis above, movement specification in the $20^{\circ}$ condition was slower relative to Experiment 1 . This was confirmed by a chi-square analysis: the observed percentage of correct responses first exceeded chance levels during the 130-179 ms S-R intervals vs. 80-129 ms in the $20^{\circ}$ condition of Experiment 1. In contrast to the data shown in Figure 14a, however, specification time was actually faster in the $80^{\circ}$ versus the $20^{\circ}$ condition. This occurred due to the large number of responses in between the target locations in the $80^{\circ}$ condition and the large spatial range across which responses were considered "correct" in this analysis $\left( \pm 40^{\circ}\right)$. It is clear from the response histograms (see Figure 9) that this less stringent accuracy criterion fails to capture the time course of movement specification in the $80^{\circ}$ condition. 


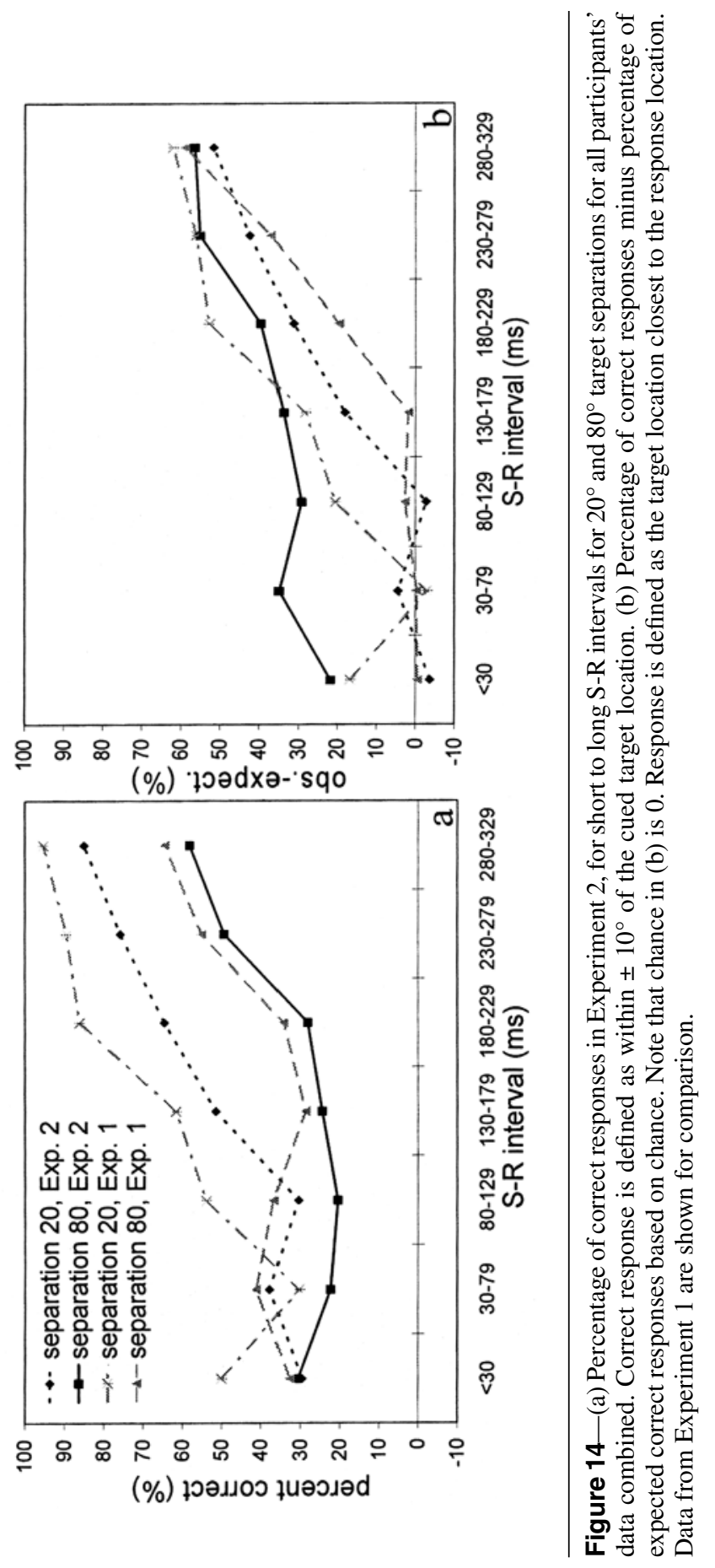




\section{Discussion}

This experiment tested the proposal that allowing on-line corrective movements in the timed-initiation paradigm would create a more distributed pattern of activation in LTM, resulting in continuous-looking motor planning even when targets were separated by more than $60^{\circ}$. Results supported this proposal. Changing the task instructions resulted in continuous movement planning both when targets were close together $\left(20^{\circ}\right)$ and far apart $\left(80^{\circ}\right)$. This supports the DFT which claims that the continuous and discrete planning modes arise from a single motor planning system and how this system integrates task, target, and LTM inputs.

The slower specification time in the $20^{\circ}$ condition of the present experiment is consistent with our proposal that manipulating the task instructions would make the LTM input more distributed (i.e., less focused). According to the DFT, it should take longer for a precise movement plan to build from a more distributed LTM input (see Erlhagen \& Schöner, 2002). The theory also predicts that such specification time effects should not be evident when the LTM input is very distributed. This explains why specification time in the $80^{\circ}$ conditions across Experiments 1 and 2 were comparable, even though responses looked discrete in Experiment 1 and continuous in Experiment 2.

These movement specification time findings are consistent with recent neural data showing faster movement specification in a precuing task as the specificity of the precue was increased. Specifically, Bastian and colleagues $(1998 ; 2003)$ recorded from an ensemble of neurons in motor cortex as two Rhesus monkeys performed a center-out pointing task. Just prior to a "go" signal, the monkeys were shown precuing information that specified one, two, or three possible adjacent targets. As the specificity of the precuing information increased, the distribution of population activity sharpened. Moreover, faster reaction times were associated with sharper population distributions. Note that these neural data mirrored simulations of the DFT (see Bastian et al., 1998; for related findings, see Erlhagen et al., 1999; Jancke et al., 1999).

\section{General Discussion}

In Experiment 1, we replicated the central findings of Ghez and colleagues (1997) using a modified task. When the target separation was small, responses looked continuous, and when the target separation was large, responses looked discrete. According to the two modes account, these results reflect the strategic use of different planning modes based on the separation of the targets (e.g., Ghez et al., 1997; Favilla, 1997; Favilla, 2002; Ottes et al., 1984). According to the DFT, however, the continuous and discrete response patterns both emerge from a single system that is "preshaped" differently by inputs at short planning times depending on the target separation. Experiment 2 attempted to distribute activation associated with one contribution to the motor planning system-a LTM input-in a condition where targets were far apart by allowing participants to correct their responses. We hypothesized that this would result in LTM traces of motor plans in between the target locations, which, according to the DFT, would result in a more continuous looking response distribution. Results were consistent with this proposal: participants initiated movements in between the target locations at short planning 
times, even when targets were far apart. Importantly, this was the case even after we controlled for corrections early in the movement. Thus, movement planning can appear continuous, even at large target separations.

A central insight of the DFT is that motor plans arise from a single task-specific and flexible planning system that integrates information about the task environment, target-specific input, and previous movement history. One implication of this on-line integration of inputs is that researchers must treat these three factors carefully, because modifications in one factor can lead to non-linear changes in the resultant motor plans (see Erlhagen \& Schöner, 2002). As a concrete example, we made a seemingly minor change to the task in Experiment 2 by allowing participants to correct their movements - a change which, at face value, should primarily affect what happens after movement initiation (see Prablanc \& Martin, 1992). The result was quite dramatic: participants' responses at movement initiation looked continuous at short planning times, even when the targets were far apart and early corrections were removed.

According to Ghez et al. (1997) the continuous and discrete modes reflect two different strategies. In this context, it is possible that our change in task instructions led to a shift in participants' strategy choice: faced with the opportunity to correct their movements, participants might have used the continuous mode in the $80^{\circ}$ condition in an attempt to improve their accuracy. For instance, in some cases, participants guessed to the center target at short planning times and then veered strongly to the left or right depending on the actual target presented. Indeed, this tendency was more pronounced when we eliminated trials with early corrections (see Figure 12). It is possible that this bias reflects some strategic guessing, that is, the strategic selection of the continuous mode even though the targets were far apart.

Although this is a viable interpretation of our results, it is an interpretation that demands further clarity regarding the concept of "strategy," the factors that influence strategy choice, and the processes that implement particular strategies. The DFT offers an explanation of how different "strategies" emerge from a neural network, and places constraints on when one strategy will emerge over another. Clearly, then, the concept of motor planning strategies is not needed to explain findings from the timed-initiation paradigm. That said, there is a sense in which the DFT does operate in different modes-averaging patterns of activation in LTM in some cases and maintaining discrete patterns of activation in others (see Figure 7). In this context, one could consider the DFT as a neurally-plausible implementation of a motor planning system capable of "strategic" responding.

It is also important to note that we are not dismissing the notion of strategic responding outright. Indeed, in recent work, we have implemented a type of "strategic" responding in dynamic field models of spatial working memory (Spencer et al., 2006; for related ideas, see Wilimzig \& Schöner, 2006, unpublished data). Specifically, by shifting the relative strengths between perceptual and LTM inputs, we effectively captured developmental changes in spatial recall versus spatial recognition responses. Importantly, the DFT forced us to specify exactly what we meant by the neural system choosing a task-specific "strategy," the factors that influenced strategy choice, and the processes that implemented particular strategies.

Although the DFT provides a useful framework for thinking about the two modes account, it is important to acknowledge that, at present, this model offers 
only a limited view of on-line corrections and how such corrections impact the planning system. Here, it is useful to place the DFT in a broader motor control context outlined by Schöner (1995). Schöner proposed that motor control is organized into three levels - a goal level, a timing level, and a load level. At the goal level, global parameters of a task are set and controlled-movement direction, movement distance, and so on. At the timing level, the specific temporal characteristics of an action are controlled. Finally, at the load level, forces generated by muscle contraction are controlled to move the actor along a desired path. Within this framework, the DFT can be thought of as a specific theory of goal-level dynamics. To account for on-line corrective movements, however, one needs to consider both what is happening at the goal level and what is happening at the level of trajectory control (i.e., at the timing and load levels).

Consider, for instance, the following example: a participant in Experiment 2 might initiate a movement toward $80^{\circ}$ and then veer off-course to the right several milliseconds into the movement, turning in response to a glimpse of the $-80^{\circ}$ target. How might the activation in the motor planning field be reflected in the details of the reaching trajectory? Recall that activation in the planning field might "jump" from $80^{\circ}$ to a location near $-80^{\circ}$, or activation at $80^{\circ}$ could "drift" from $80^{\circ}$ through intervening target directions, finally settling in on a direction near $-80^{\circ}$. As discussed earlier, the work on mental rotation supports a continuous rotation of the motor plan for a range of target separations; however, even wider target separations may result in the motor plan "jumping" rather than "drifting." Regardless of whether the movement plan jumps or drifts, the trajectory level dynamics will be necessarily constrained to move smoothly through spatial locations in between $0^{\circ}$ and $80^{\circ}$ en route to the newly planned direction. This highlights that there might not be a one-to-one match between the goal level and the details of the trajectory.

The lack of one-to-one correspondence between goal and trajectory level dynamics makes it difficult to tease apart the unique contributions of each level. Nevertheless, we contend that this is an empirically tractable problem in at least two ways. First, jumping versus drifting activation peaks in the DFT should produce unique signatures at the trajectory level, signatures which are consistent with theories that posit "via points" or "via postures" (e.g., Flash \& Henis, 1991; Rosenbaum et al., 1995). In particular, perturbations of the trajectory in the direction of $80^{\circ}$ should not be counteracted in the case where activation jumps from $0^{\circ}$ to $80^{\circ}$ in the DFT. By contrast, the same perturbations should be counteracted in the case of a drifting activation profile. Second, jumping versus drifting activation peaks should leave unique memory traces, traces which can be probed from trial to trial. For instance, if activation jumps from $0^{\circ}$ to $80^{\circ}$ on a particular planning trial, there should be a stronger facilitation of the $80^{\circ}$ location on the next trial relative to a case where activation drifts through intervening directions. In this latter case, there should be some facilitation of locations in between $0^{\circ}$ and $80^{\circ}$. We contend that long-term memory traces offer a new window into the subtle interplay between goal-level and trajectory-level dynamics that has been relatively unexplored in the planning literature.

Beyond these empirical insights, the DFT offers an important general theoretical insight: behavioral evidence showing a difference in performance in different situations does not necessarily indicate the operation of separate planning systems (for related examples, see Van Orden et al., 2001; Munakata, 2001; Spencer \& 
Schutte, 2004). There has been a growing tendency in the motor control literature to fractionate the motor control system into separable systems (e.g., Goodale \& Haffenden, 1998; Goodale et al., 2004). Although we certainly agree that analysis of the motor control system sometimes requires discussion of unique contributions to movement responses (see Schöner, 1995), decomposition of a system into parts or levels must be done in a way that considers integration as well, that is, one must seriously consider how the "parts" of the system work together to produce an integrated motor behavior. The DFT provides a concrete example of a model that takes integration seriously. As evidence, recent work in autonomous robotics demonstrates that dynamic fields can be used to handle the details of motor planning in a way that allows for real-time coupling to sensorimotor processes (see Bicho et al., 2000).

\section{Acknowledgments}

This research was supported by NIMH grants RO1 MH62480 and NSF BCS 00-91757 awarded to John P. Spencer. We would like to thank Dr. Gregor Schöner for his valuable comments on this manuscript, and the late Dr. Esther Thelen for intellectual support during the completion of Experiment 2. We would also like to thank Dr. Wolfram Erlhagen for discussions of the dynamic field theory, and Dr. Alycia Hund, Regan Vinton, Phillippe Taborga, and numerous undergraduates for their help with data collection and analysis.

\section{References}

Bastian, A., Riehle, A., Erlhagen, W., \& Schöner, G. (1998). Prior information preshapes the population representation of movement direction in motor cortex. Neuroreport, 9, 315-319.

Bastian, A., Schöner, G., \& Riehle, A. (2003). Preshaping and continuous evolution of motor cortical representations during movement preparation. European Journal of Neuroscience, 18, 2047-2058.

Bicho, E., Mallet, P., \& Schöner, G. (2000). Target representation on an autonomous vehicle with low-level sensors. International Journal of Robotics Research, 19, 424-427.

Chou, I., Sommer, M.A., \& Schiller, P.H. (1999). Express averaging saccades in monkeys. Vision Research, 39, 4200-4216.

Dounskaia, N., Swinnen, S.P., \& Walter, C.B. (2000). Principle of control of rapid multijoint movements: The leading joint hypothesis. In J.M. Winter \& P.E. Crago (Eds.) Biomechanics and Neural Control of Posture and Movement. New York: Springer-Verlag.

Erlhagen, W., Bastian, A., Jancke, D., Riehle, A., \& Schöner, G. (1999). The distribution of neuronal population activation (DPA) as a tool to study interaction and integration in cortical representations. Journal of Neuroscience Methods, 94, 53-66.

Erlhagen, W., \& Schöner, G. (2002). Dynamic Field Theory of movement preparation. Psychological Review, 109, 545-572.

Favilla, M. (1997). Reaching movements: concurrency of continuous and discrete programming. Neuroreport, 8, 3973-3977.

Favilla, M., (2002). Reaching movements: mode of motor programming influences programming time by itself. Experimental Brain Research, 144, 414-418.

Favilla, M., Gordon, J., Hening, W., \& Ghez, C. (1990). Trajectory control in targeted force impulses: VII. Independent setting of amplitude and direction in response preparation. Experimental Brain Research, 79, 530-538.

Feldman, A.G., \& Levin, M.F. (1995). The origin and use of positional frames of reference in motor control. Behavioral and Brain Sciences, 18, 723-806. 
Flash, T., \& Henis, E. (1991). Arm trajectory modifications during reaching towards visual targets. Journal of Cognitive Neuroscience, 3, 220-230.

Georgopoulos, A.P., Lurito, J.T., Betrides, M., Schwartz, A.B., \& Massey, J.T. (1989). Mental rotation of the neuronal population vector. Science, 243, 234-236.

Georgopoulos, A.P., \& Massey, J.T. (1987). Cognitive spatial-motor processes. 1. The making of movements at various angles from a stimulus direction. Experimental Brain Research, 65, 361-370.

Ghez, C., Favilla, M., Ghilardi, M.F., Gordon, J., Bermejo, R., \& Pulman, S. (1997). Discrete and continuous planning of hand movements and isometric force trajectories. Experimental Brain Research, 115, 217-233

Goodale, M.A., \& Haffenden, A. (1998). Frames of reference for perception and action in the human visual system. Neuroscience \& Biobehavioral Reviews, 22, 161-172.

Goodale, M.A., Westwood, D.A., \& Milner, A.D. (2004). Two distinct modes of control for object-directed action. Progress in Brain Research, 144, 131-144.

Hening, W., Vicario, D., \& Ghez, C. (1988). Trajectory control in targeted force impulses. IV. Influences of choice, prior experience and urgency. Experimental Brain Research, 71, 103-115.

Jancke, D., Erlhagen, W., Dinse, H.R., Akhavan, A.C., Giese, M., Steinhage, A., \& Schöner, G. (1999). Parametric population representation of retinal location: neuronal interaction dynamics in cat primary visual cortex. Journal of Neuroscience, 19, 9016-9028.

Munakata, Y. (2001). Graded representations in behavioral dissociations. Trends in Cognitive Science, 5, 309-315.

Ottes, F.P., Van Gisbergen, A.M., \& Eggermont, J.J. (1984). Metrics of saccade responses to visual double stimuli: two different modes. Vision Research, 24, 1198-1179.

Prablanc, C., \& Martin, O. (1992). Automatic control during hand reaching at undetected two-dimensional target displacements. Journal of Neurophysiology, 67, 455-469.

Rosenbaum, D.A., Loukopoulos, L.D., Meulenbroek, R.G.J., Vaughan, J., \& Engelbrecht, S.E. (1995). Planning reaches by evaluating stored postures. Psychological Review, 102, 28-67.

Schöner, G. (1995). Recent developments and problems in human movement science and their conceptual implications. Ecological Psychology, 7, 291-314.

Schöner, G., Kopecz, K., \& Erlhagen, W. (1997). The dynamic neural field theory of motor programming: Arm and eye movements. In P.G. Morasso \& V. Sanguineti (Eds.), Selforganization, computational maps and motor control, Psychology Series, Vol. 119 (pp. 271-310). North Holland: Elsevier.

Schutte, A.R., Spencer, J.P., \& Schöner, G. (2003). Testing the dynamic field theory: working memory for locations becomes more spatially precise over development. Child Development, 74, 1393-1417.

Spencer, J.P., \& Hund, A.M. (2002). Prototypes and particulars: Geometric and experience-dependent spatial categories. Journal of Experimental Psychology: General, 131, 16-37.

Spencer, J.P., \& Schöner, G. (2003). Bridging the representational gap in the dynamic systems approach to development. Developmental Science, 6, 392-412.

Spencer, J.P., Schutte, A.R. (2004). Unifying representations and responses: Perseverative biases arise from a single behavioral system. Psychological Science, 15, 187-193.

Spencer, J.P., Simmering, V., \& Schutte, A.R. (2006). Toward a formal theory of flexible spatial behavior: Geometric category biases generalize across pointing and verbal response types. Journal of Experimental Psychology: Human Perception and Performance, 32, 473-490.

Spencer, J.P., Simmering, V.R., Schutte, A.R., \& Schöner, G. (in press). What does theoretical neuroscience have to offer the study of behavioral development? Insights from a dynamic field theory of spatial cognition. In J. Plumert \& J.P. Spencer (Eds.) The Emerging Spatial Mind. Oxford University Press. 
Thelen, E., Corbetta, D., \& Spencer, J.P. (1996). Development of reaching during the first year: Role of movement speed. Journal of Experimental Psychology: Human Perception and Performance, 22, 1059-1076.

Van Orden, G.C., Pennington, B.F., \& Stone, G.O. (2001). What do double dissociations prove? Cognitive Science, 25, 111-172.

Van Sonderen, J.F., Gielen, C.C., \& van der Gon Denier, J.J. (1989). Motor programmes for goal-directed movements are continuously adjusted according to changes in target location. Experimental Brain Research, 78, 139-146.

Wilimzig, C., \& Schöner, G. (2006). A dynamic field theory of categorical and continuous response generation. Manuscript in preparation.

Winter, D.A. (1990). Biomechanics and motor control of human movement, 2nd ed. New York: John Wiley \& Sons.

\section{Endnotes}

1. All simulations were conducted in MATLAB (Mathworks, Inc.; the MATLAB code is available from the authors on request). The MP field equation (see Erlhagen and Schöner, 2002) was integrated using the Euler method. A network with 251 neurons was used for all simulations. Each run of the model simulated a single trial in the timed-initiation paradigm, with 1 time step = $1 \mathrm{~ms}$. Trials began with the MP field initialized at a negative resting level of -3 units. At the "go" signal (which occurred simultaneously with target offset), the resting level of the MP field was raised to -1 to allow a peak to be created in the field. The task input remained "on" throughout the trial, while the target input appeared and disappeared for a short duration as in the timed-initiation paradigm (i.e., 30-300 ms). The long-term memory dynamics were engaged at the start of each trial. The activation pattern in LTM at the start of each trial was set to the activation pattern in LTM that emerged at the end of the just-previous trial. Thus, there was no decay in LTM in between trials. After the "go" signal, we continued the simulation for $750 \mathrm{~ms}$. The model's response was determined by finding the movement direction associated with the maximum activation at this point in time. 